\title{
Mesurer et spatialiser la connectivité pour modéliser les changements des systèmes environnementaux. Approches comparées en écologie du paysage et en géomorphologie
}

\section{Measuring and spatializing connectivity within environmental systems networks to predict changes. Comparative approaches in landscape ecology and geomorphology}

\author{
Marc Bourgeois ${ }^{*}$, Étienne Cossart ${ }^{\mathrm{a}}$, Mathieu Fressard ${ }^{\mathrm{b}}$ \\ a Université de Lyon, Université Jean Moulin Lyon 3, UMR 5600 CNRS-Environnement Ville Société - 18 rue Chevreul, 69007 Lyon, France. \\ ${ }^{\mathrm{b}}$ Université de Lyon, Lumière (Lyon 2), UMR Environnement Ville Société 5600, CNRS - Bâtiment Europe, 5 avenue Pierre Mendès-France, 69676 Bron cedex, France.
}

INFORMATION SUR L'ARTICLE

Article soumis le 18 juin 2017

Reçu sous sa forme révisée le 12 janvier 2018.

Définitivement accepté le 16 janvier 2018.

${ }^{*}$ Auteur correspondant : Tél : +33 (0)4 78787462 marc.bourgeois@univ-lyon3.fr (M. Bourgeois) etienne.cossart@univ-lyon3.fr (É. Cossart) mathieu.fressard@univ-lyon2.fr (M. Fressard)

\begin{abstract}
RÉSUMÉ
Le concept de connectivité est mobilisé dans plusieurs disciplines relevant des sciences de l'environnement, comme la géomorphologie ou l'écologie du paysage, pour mieux évaluer la réponse des systèmes environnementaux aux forçages naturels (e.g. climatiques) et anthropiques (e.g. changements de modes d'occupation du sol, construction d'infrastructures linéaires, etc.). La mesure et la spatialisation de la connectivité permet en effet de comprendre aussi bien l'organisation des réseaux de flux sédimentaires (géomorphologie) que des réseaux écologiques (écologie du paysage), notamment pour mettre en évidence dans quelle mesure ces réseaux sont aptes à propager des perturbations. Bien que les objets d'étude soient différents, nous pouvons cependant identifier des passerelles méthodologiques communes entre ces deux champs thématiques, notamment pour spatialiser les propriétés des réseaux écologiques et de flux sédimentaires à l'aide de la théorie des graphes. La représentation topologique d'un réseau sous forme de graphes constitue un support permettant de mesurer la connectivité via le calcul de métriques à différentes échelles. Pour illustrer nos propos, nous proposons ici de comparer deux exemples de protocoles méthodologiques, l'un visant à évaluer l'impact écologique de différentes formes d'urbanisation sur l'aire urbaine de Besançon, et l'autre visant à évaluer l'impact du changement climatique sur un réseau de flux sédimentaires dans un bassin versant alpin. Nous montrons au final que dans le champ de la géomorphologie ou de l'écologie du paysage, l'analyse de la connectivité constitue un socle méthodologique pertinent pour mieux évaluer les propriétés de résilience de systèmes environnementaux face à des forçages globaux (changements climatiques) ou plus locaux (activités anthropiques).
\end{abstract}

Mots clés : connectivité, écologie, réseaux, paysage, théorie des graphes, flux sédimentaires, géomorphologie.

\section{ABSTRACT}

Connectivity is a key concept used in several disciplines, such as geomorphology or landscape ecology, to better assess the response of environmental systems to external forces (e.g. climate change or anthropogenic influences). Some metrics of connectivity provides the possibility to understand how sedimentary fluxes (geomorphological networks) and ecological networks (landscape ecology) are spatially organized. We propose in this paper a comparative approach in these two disciplines: especially comparison of metrics that describes connectivity. Although the studied systems are different, we can identify common methodological frames. The properties of ecological networks and sedimentary fluxes can be described through graph theory. The topological pattern of a network through graphs constitutes a first step to measure connectivity patterns by calculating metrics at different scales. We first present an example of a methodological protocol regarding landscape ecology: the example seeks at evaluating the ecological impact of different patterns of urbanization. Second, we present an example focusing on geomorphology, to describe the geomorphic evolution of a catchment in a global warming context. Finally, from a comparison between the two case studies, we show that, in both fields of geomorphology and landscape ecology, the analysis of connectivity constitutes a relevant methodological basis to evaluate the resilience properties of environmental systems in a changing environment.

Keywords: connectivity, ecology, network, landscape, graph theory, sedimentary flows, geomorphology.

\section{Introduction}

La connectivité est un concept clef actuellement largement mobilisé dans les sciences de l'environnement, plus particulièrement dans le cadre des débats relatifs au changement global. Il s'agit par exemple de mieux comprendre comment les systèmes environnementaux répondent aux changements climatiques ou encore aux forçages anthropiques. L'analyse de la connectivité s'applique d'une façon générale à des réseaux et donc à des systèmes environnementaux qui peuvent être formalisés sous forme de réseaux d'interactions (Taylor et al., 1993 ; Heckmann et al., 2015 ; Cossart et Fressard, 2017). L'un des principaux problèmes scientifiques actuellement posé par l'étude de ces réseaux d'interactions est leur propriété 
d'auto-organisation (Bak et al., 1987, Bak et al., 1988), et plus particulièrement l'existence de mécanismes par lesquels le même stimulus peut faire émerger dans le comportement global du système à la fois des événements de faible et de haute magnitude. L'hypothèse sous-jacente est que les systèmes étudiés sont caractérisés par une variabilité interne, reflétant les qualités de connexion entre les différentes parties du système. De la combinaison de ces connexions émerge un comportement non linéaire du système, qui s'avère complexe à anticiper. Il s'agit dès lors de comprendre comment s'instaurent des équilibres et/ou des déséquilibres dans ces systèmes, de mesurer si ces systèmes sont aptes à transférer des perturbations extérieures, et donc de documenter leur capacité de résilience. Décrypter la connectivité et décrire les propriétés des réseaux d'interactions au sein des systèmes environnementaux nécessite toutefois le développement de protocoles méthodologiques spécifiques qui mettent le plus souvent l'espace, en tant que support explicatif, au cœur de la démarche (Cossart et Fressard, 2017).

L'objectif de cet article est de montrer qu'une analyse géographique peut être féconde pour décrypter ce qui, dans le fonctionnement d'un système environnemental, relève d'une auto-organisation interne et d'un forçage externe. Pour cela nous abordons une démarche comparative entre deux champs complémentaires parmi les sciences de l'environnement : l'écologie du paysage et la géomorphologie. Il s'agit de mettre en évidence les étapes communes d'évaluation, de spatialisation et d'interprétation de la connectivité dans ces deux champs. Une telle confrontation interdisciplinaire permet de montrer que si les verrous méthodologiques ne se situent pas dans les mêmes phases du protocole propre à chaque discipline, ces verrous peuvent être levés en s'inspirant des innovations développées dans le champ thématique connexe.

\section{2. État de l'art}

\subsection{Historique des études portant sur la connectivité}

Les études portant sur les réseaux d'interactions sont utilisées de longue date dans le champ de l'environnement, et ont été pionnières en écologie pour décrire les relations trophiques entre les espèces (Hardy, 1924) et étudier les conséquences de cette connectivité trophique sur la stabilité des écosystèmes (May, 1973). Dans le prolongement de ces travaux, le concept de connectivité a été plus récemment utilisé pour évaluer la cohérence spatiale d'un système, une cohérence nécessaire pour maintenir ou restaurer les fonctions écologiques (Taylor et al., 1993 ; Bennett, 2004). Dans le champ de l'archéologie, la connectivité est également utilisée comme une clef de compréhension des modalités de l'organisation globale de systèmes sociaux ou économiques (Östborn et Gerding, 2014), par exemple pour favoriser l'anticipation des aléas et le partage d'information à longues distances chez les chasseurs-cueilleurs (Whallon, 2006). Elle fournit des clefs d'interprétation en termes d'évolution des sociétés humaines, ainsi que du développement de la coopération chez les chasseurs-cueilleurs (Apicella et al., 2012).

En parallèle, des travaux ont davantage cherché à décomposer le fonctionnement global des réseaux d'interactions, à procéder à une descente d'échelle d'analyse de leur fonctionnement. Fondées sur la théorie des graphes, des métriques ont été développées avec pour objectif d'identifier des points névralgiques où tout changement peut avoir un impact sur l'ensemble du système, et ce, qu'il s'agisse de réseaux de transport (Cole et King, 1968 ; Gleyze, 2008), de réseaux sociaux (Freeman, 1979) ou, plus récemment, de réseaux écologiques (Belisle, 2005). Dans ces études, l'objectif est de fournir une hiérarchie de l'influence des nœuds du réseau, et de montrer que les nœuds caractérisés par une connectivité élevée ont une influence considérable sur le fonctionnement global du réseau. En effet, ces nœuds à forte connectivité sont des articulations traversées par le plus grand nombre de chemins possibles dans le réseau : ils contrôlent donc une grande part des flux qui animent le système. En corollaire, de tels nœuds à connectivité élevée sont ceux où une perturbation entraînerait des perturbations maximales dans le fonctionnement global du réseau (Haggett et Chorley, 1969 ; Gleyze, 2008 ; Newman, 2010).

Les questionnements sont finalement similaires pour les géographes-géomorphologues, qui ont mobilisé le concept pour décrypter l'instabilité spatio-temporelle des signaux sédimentaires dans les bassins-versants (Hoffmann, 2015) et comprendre le caractère anarchique des flux sédimentaires mis en évidence il y a trois décennies (Ferguson, 1981). L'une des hypothèses est en effet que des combinaisons locales, ayant cours au niveau de points névralgiques, sont à l'origine de perturbations globales du réseau formé par des flux sédimentaires dans un bassin versant. Toutefois ces études restent en développement, entravées notamment par la difficulté à bâtir des métriques dans des graphes orientés, dirigés par la gravité (Rodrigue, 2017). En effet, les indices fournis par la théorie des graphes ont été principalement appliqués à des graphes non orientés, particulièrement en géographie (Pumain et SaintJulien, 2010).

Cet historique montre, en écologie comme en géographie, que la connectivité aide à synthétiser la complexité du système étudié et de son fonctionnement : il s'agit de comprendre comment la combinaison d'interactions élémentaires (au sein d'une chaîne trophique, au sein d'une cascade hydro-sédimentaire) fait émerger un signal, un comportement original (et difficile à anticiper) de l'ensemble du réseau.

\subsection{La connectivité des écosystèmes}

Les écosystèmes sont le support de divers processus écologiques comme l'évolution des espèces, les relations trophiques entre les espèces, le mutualisme ou encore la compétition interspécifique. L'identification et la caractérisation de ces processus relève principalement du domaine de l'écologie. Les méthodes issues de l'écologie du paysage permettent de simplifier certains de ces processus en les représentants spatialement dans la mosaïque paysagère (Forman et Godron, 1986). Ce modèle relève d'une vision zénithale du paysage et se compose de taches et de corridors (fig. 1). Les espaces interstitiels correspondent à la matrice paysagère. Les taches sont des éléments compacts, non linéaires et homogènes dont la nature est différente de la matrice. Les corridors sont des bandes dont la nature diffère de la matrice présente de part et d'autre. Ils peuvent avoir plusieurs fonctions : conduit, habitat, filtre, barrière, source et puits (Hess et Fischer, 2001).

La connectivité du paysage (ou connectivité paysagère) est définie comme le degré par lequel le paysage facilite ou influence les mouvements entre les taches d'habitat préférentiel pour une espèce donnée (Taylor et al., 1993). La connectivité dépend à la fois de la configuration spatiale du paysage et de la manière dont les individus interagissent dans cette structure spatiale (Wiens et al., 1997). Merriam (1984) distingue deux types de connectivité : la connectivité structurelle, correspondant à l'agencement des structures spatiales d'un paysage (fig. 2A), et la connectivité fonctionnelle, correspondant à l'influence des structures paysagères sur les organismes. La connectivité fonctionnelle se distingue ellemême en connectivité fonctionnelle réelle (fig. 2B) et en connectivité fonctionnelle potentielle (Calabrese et Fagan, 2004) (fig. 2C). La connectivité fonctionnelle réelle est estimée sur le terrain par observation de certaines espèces alors que la connectivité 


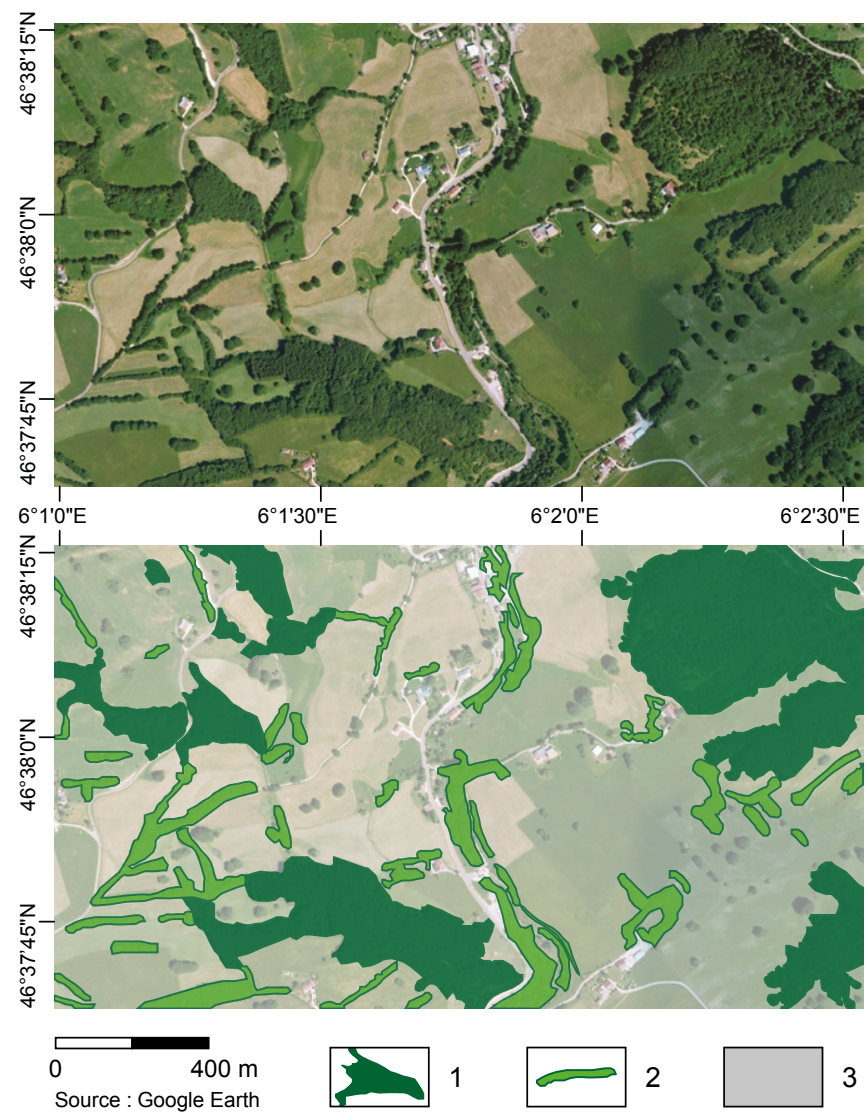

Fig. 1 - Modèle de la mosaïque paysagère.

Commune de Foncine-le-Bas (Jura), d'après Forman et Godron (1986), et Bourgeois (2015). A : Vue zénithale du paysage ; B : Application du modèle matrice-tachecorridor. 1. Tache; 2. Corridor; 3. Matrice

\section{Fig. 1 - Patch-corridor-matrix model.}

Municipality of Foncine-le-Bas (Jura, France). Adapted from Forman and Godron (1986), and Bourgeois (2015). A: Zenithal view of the landscape; B: Application of the patch-corridor-matrix model. 1. Patch; 2. Corridor; 3. Matrix.

fonctionnelle potentielle est estimée à partir de modèles de simulation spatiale (Tischendorf et Farig, 2000). Pour une espèce donnée, la connectivité fonctionnelle dépend des caractéristiques de la mosaïque paysagère : d'une part les taches d'habitat, et d'autre part la composition de la matrice entre ces taches. La matrice peut favoriser les déplacements de l'espèce, par la présence de corridors ou d'éléments d'occupation du sol favorables. Inversement, elle peut aussi limiter les déplacements avec les éléments d'occupation du sol défavorables. L'étude du processus de dispersion permet de caractériser les déplacements des individus parmi les populations (Ims et Yoccoz, 1997). Ce processus est essentiel à la survie des populations puisqu'il permet de maintenir la diversité génétique et de limiter le déclin des populations. La dispersion des individus entre leurs taches d'habitat permet aux espèces de coloniser de nouvelles taches d'habitat à travers la dynamique des métapopulations (Hanski et Gilpin, 1991). Le processus de dispersion est implicitement lié au concept de connectivité paysagère. Dans la mosaïque paysagère, les taches d'habitat d'une espèce ou d'un groupe d'espèces donné sont dites connectées lorsque les flux de dispersion se produisent ou peuvent se produire de manière régulière entre ces taches. Inversement, les taches d'habitat sont déconnectées si elles n'échangent aucun flux de dispersion. La connectivité paysagère dépend des caractéristiques de la mosaïque paysagère ainsi que des comportements, des préférences d'habitat et des capacités de dispersion de l'espèce considérée (Johnson et

A

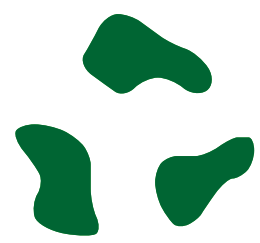

Forte connectivité (taches rapprochées)

High connectivity (close patches)
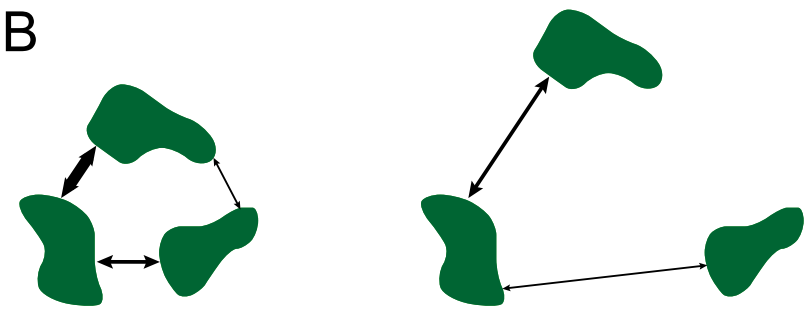

Flux de dispersion observés pour une espèce donnée Observed dispersal flux for a given species

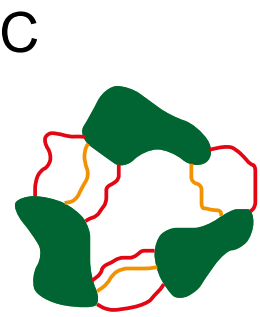

Taches potentiellement connectées pour les deux espèces

Potentially connected patches for both species

Faible connectivité (taches éloignées) Low connectivity (distant patches)

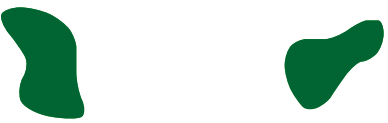


une barrière pour les déplacements d'un certain nombre d'espèces. Dans ce cas, la connectivité fonctionnelle de la matrice paysagère est réduite. Les mesures de connectivité peuvent alors permettre de quantifier de manière objective ces différentes perturbations, un objectif scientifique que l'on retrouve également dans le champ de la géomorphologie.

\subsection{La connectivité sédimentaire}

Les apports du concept de connectivité en géomorphologie restent en phase de développement, compte-tenu de la difficulté de formaliser les réseaux qui sous-tendent les flux de sédiments à la surface de la Terre. Les travaux sur la connectivité sédimentaire s'inspirent toutefois largement des réflexions menées par des hydrologues montrant que l'agencement spatial des connexions hydrologiques explique la nature du signal hydrologique à l'exutoire d'un bassin versant (Delahaye et al., 2002 ; Douvinet et al., 2008).

Nous reprenons ici la terminologie de Heckmann et Schwanghart (2013), distinguant le couplage, qui est un phénomène local, de la connectivité, qui est une propriété globale. Selon eux «le lien qui assure la relation entre deux unités géomorphologiques par le transport de sédiments est appelé couplage ». Dans la lignée des travaux de Harvey (2001) ou Faulkner (2008), le couplage se réfère donc aux interactions élémentaires à une échelle locale. En complémentarité, "l'assemblage des différents couplages élémentaires, l'effet combiné des interactions latérales (du versant au talweg) et longitudinales (d'amont en aval d'un hydrosystème) entre les composants du système, est appelé connectivité (sédimentaire)" (Heckmann et Schwanghart, 2013). Même si les travaux portant sur la connectivité hydro-sédimentaire sont en plein essor, les définitions restent toutefois peu stabilisées. Pourtant, identifier et comprendre les agencements de flux sédimentaires à l'intérieur des bassins-versants revêt de multiples enjeux : (i) ces flux enregistrent les changements climatiques et les impacts créés par l'homme à la surface terrestre ; (ii) les flux sédimentaires sont en prise avec la ressource en sol et les formations superficielles, qu'ils sont susceptibles d'appauvrir ou d'enrichir ; (iii) les flux sédimentaires modifient le fonctionnement des cours d'eau et plus particulièrement les écosystèmes qu'ils représentent. Les enjeux sont nombreux, stimulant les recherches sur le comportement érosif. Les flux sédimentaires peuvent en effet être complexes, au sens de la théorie de la complexité : étroitement dépendants de multiples couplages locaux, ils restent difficilement prévisibles, à tel point que plusieurs auteurs y voient une sedimentological anarchy (Walker, 1990 ; Hoffmann, 2015). Pour comprendre cette complexité, l'enjeu est d'ouvrir les boîtes noires qui demeurent à l'intérieur des réseaux de flux sédimentaires. Les auteurs insistent alors sur la nécessité d'explorer les couplages locaux, afin de comprendre comment et pourquoi ils activent ou bloquent, alternativement, les filiations de processus dans une cascade sédimentaire. Il s'agit alors de mesurer la qualité de la connectivité sédimentaire, c'est-à-dire l'aptitude d'un réseau à transmettre les sédiments de l'amont vers l'aval, en identifiant les éventuels points de blocage.

La notion de connectivité, largement utilisée dans des travaux de recherche récents en géomorphologie et en écologie du paysage, a donc été mobilisée pour permettre une meilleure compréhension de systèmes environnementaux complexes. Bien que les objets de recherche soient différents, plusieurs transversalités peuvent être observées entre ces deux disciplines et l'analyse de la connectivité offre un cadre conceptuel et méthodologique commun.

Le premier objectif de cet article est de faire un parallèle entre les approches développées en écologie du paysage et en géomorphologie pour spatialiser et mesurer la connectivité. Dans un second temps, deux cas d'applications seront développés pour chacun des domaines d'études : (i) pour l'écologie du paysage, un protocole méthodologique visant à évaluer l'impact écologique de différents formes d'urbanisation dans l'aire urbaine de Besançon ; (ii) pour la géomorphologie, un protocole méthodologique visant à évaluer l'évolution spatio-temporelle de la connectivité sédimentaire dans un bassin-versant du massif des Écrins. De là découle un troisième objectif : identifier des approches permettant un enrichissement mutuel entre ces deux disciplines, amenant à lever certains verrous méthodologiques.

\section{Représenter et mesurer la connectivité}

La spatialisation de la connectivité est la première étape de la démarche. Pour l'écologue du paysage, spatialiser la connectivité peut permettre l'identification de réseaux écologiques dans le cadre de mise en place de politiques de conservation, ou de recommandations à destination des aménageurs. Pour le géomorphologue, cette spatialisation peut aider à formaliser des réseaux sédimentaires dans un bassin-versant. Selon la discipline, les objets d'études sont différents. L'écologue du paysage s'intéresse plus particulièrement aux flux de déplacements d'organismes vivants comme par exemple des espèces animales tandis que le géomorphologue, lui, s'intéressera plus particulièrement à des flux de sédiments dans un système donné.

La deuxième étape est celle de la mesure de la connectivité. Pour mesurer la connectivité paysagère, différents indices, appelés métriques de connectivité, ont été développés. Les résultats issus des calculs de ces métriques peuvent servir d'aide à la décision dans le cadre de la mise en place de projets d'aménagement du territoire ou de mesures de conservation des espèces (Calabrese et Fagan, 2004). Des métriques similaires peuvent être utilisées en géomorphologie pour mettre en exergue les zones les plus sensibles d'un réseau sédimentaire, c'est-à-dire celles où tout changement entrera particulièrement en résonnance avec l'ensemble de la cascade sédimentaire.

En géomorphologie, cette analyse correspond à la recherche des «points névralgiques » des systèmes érosifs. En effet, Cossart et Fressard (2017) montrent que les points qui jalonnent les flux sédimentaires n'ont pas tous la même importance : la localisation de ces points névralgiques n'est pas neutre et, sans que cela soit encore modélisé, il apparaît un "effet papillon». Quelques variations mineures au niveau de ces points peuvent faire varier de façon significative l'ampleur des flux sédimentaires dans le système.

La troisième étape consiste à évaluer les impacts des modifications des systèmes environnementaux sur la connectivité. Les mesures de connectivité, a fortiori si elles sont réalisées de manière diachronique peuvent permettre de mesurer la perte de connectivité imputable à un changement environnemental (construction de grandes infrastructures ou de bâtiments, changements de modes d'occupation du sol). Ces mesures de connectivité peuvent être effectuées à un niveau global (un écosystème, un bassin-versant) ou à un niveau plus local (une zone humide, une tête de bassin sédimentaire). Dans certains cas, ces études d'impacts peuvent venir appuyer des processus d'aide à la décision, à destination des aménageurs ou de décideurs locaux (fig. 3A).

Depuis les années 2000, un nombre croissant de travaux en écologie du paysage utilisent la théorie des graphes pour évaluer la connectivité potentielle des paysages (Urban et Keitt, 2001 ; Galpern et al., 2011). Les graphes paysagers permettent de modéliser les réseaux écologiques des espèces à une échelle intergénérationnelle entre leurs domaines vitaux et d'analyser ainsi la 

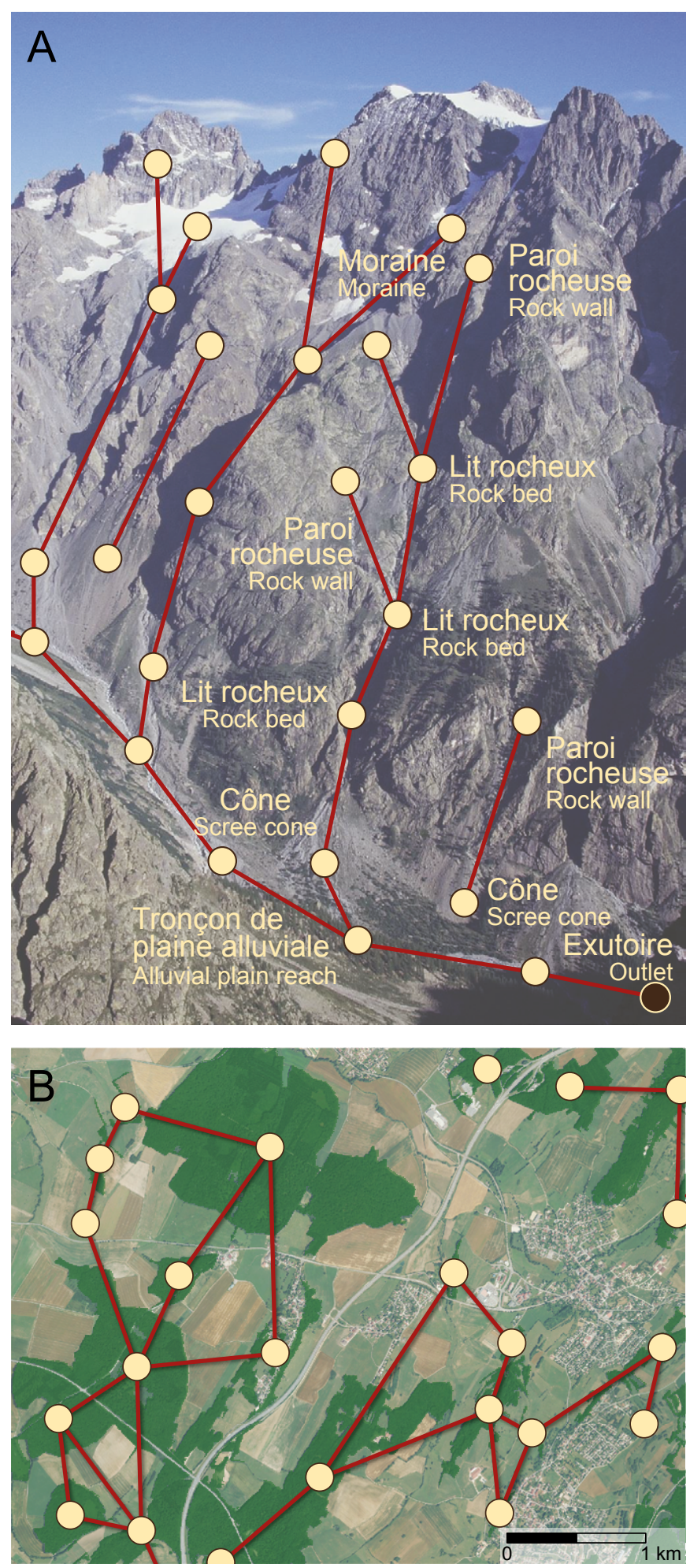

Source : BD Ortho IGN 2010
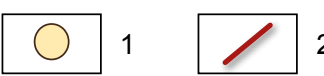

2

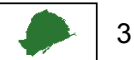

Fig. 3 - Exemple de constructions de graphes en géomorphologie et en écologie du paysage.

A : Représentation sous forme de graphes d'un réseau de flux sédimentaires (vue oblique) ; B : Représentation sous forme de graphes du réseau écologique d'une espèce forestière (vue zénithale). 1. Nœud ; 2 . Lien ; 3 . Tache d'habitat.

Fig 3 - Example of graph formalization in geomorphology and in landscape ecology.

A: Graph representation of a sedimentary fluxes network (oblic view); B: Graph representation of an ecological species network (zenithal view). 1. Node; 2. Link; 3. Habitat patch. connectivité fonctionnelle des paysages à l'échelle régionale. À ce titre, la modélisation des graphes paysagers est reconnue comme un outil pertinent d'aide à la gestion paysagère (Bunn et al., 2000 ; Dale et Fortin, 2010). Les graphes paysagers représentent la mosaïque paysagère de manière topologique (fig. 3B). Les sommets (ou "nœuds ") représentent les taches d'habitat d'une espèce ou d'un groupe d'espèces. Les déplacements potentiels entre les taches d'habitat sont symbolisés par les arêtes (ou "liens ») du graphe représentant les déplacements liés au processus de dispersion. La création d'un lien entre deux taches d'habitat suppose que ces taches soient potentiellement connectées. Afin de mieux visualiser un graphe, il est possible de le seuiller à la distance de dispersion de l'espèce étudiée. Dans ce cas, deux taches d'habitat sont connectées dans le graphe si la distance les séparant est inférieure à la distance choisie. Il s'agit généralement de la distance moyenne ou maximale de dispersion. Parmi l'ensemble des méthodes existantes pour identifier les connexions potentielles entre les taches d'habitat, l'analyse des chemins de moindre coût et la théorie des circuits sont les plus utilisées dans les travaux scientifiques. L'attribution de coûts de déplacements à chaque classe d'occupation du sol pour une espèce donnée permet d'identifier des chemins de moindre coût entre les taches d'habitat (Adriaensen et al., 2003). Ces chemins représentent les déplacements potentiels des espèces à travers la matrice paysagère. La théorie des circuits fait, elle, l'analogie entre la connectivité fonctionnelle du paysage et les flux présents dans un circuit électrique (McRae et al., 2008). Les flux de dispersion des animaux sont représentés comme des circuits électriques permettant de mettre en évidence la fréquence des parcours potentiellement empruntés par les espèces dans la mosaïque paysagère.

Plusieurs étapes sont nécessaires à la construction d'un graphe paysager : (i) cartographier le paysage d'une espèce, (ii) définir les taches d'habitat de l'espèce (nœuds du graphe), (iii) définir les liens du graphe et (iv) seuiller les liens du graphe (optionnel). La construction d'un graphe paysager permet ainsi de spatialiser la connectivité dans le paysage et de rendre possible sa représentation cartographique. En revanche, cette représentation ne permet pas toujours de comparer les graphes entre eux car il est difficile d'identifier les nœuds et les liens différents entre les graphes. Il est donc préférable de comparer des graphes en calculant des métriques de connectivité, permettant de quantifier ces différences, plutôt que de les identifier visuellement.

Pour mesurer la connectivité fonctionnelle à l'aide de graphes paysagers, certaines métriques ont été développées spécifiquement pour l'analyse des réseaux écologiques (Dale et Fortin, 2010 ; Galpern et al., 2011 ; Rayfield et al., 2011). Elles permettent de caractériser les flux potentiels de déplacements entre deux taches d'habitat en fonction de la distance qui les sépare. Certaines métriques comme Probability of Connectivity (PC) (Saura et Pascual-Hortal, 2007) permettent de caractériser la somme de ces interactions pour l'ensemble du graphe paysager.

Pour l'évaluation de la connectivité fonctionnelle des paysages, les graphes paysagers offrent un bon compromis entre leur difficulté de mise en œuvre et la réalité écologique qu'ils représentent (Calabrese et Fagan, 2004). Des études ont en effet montré que les analyses menées à partir des graphes paysagers d'une espèce donnée peuvent produire des résultats similaires à l'exploitation des résultats issus de simulations de déplacements individuels de la même espèce (Minor et Urban, 2007 ; Lookingbill et al., 2010).

Ainsi, la connectivité fonctionnelle peut être évaluée par des mesures empiriques (connectivité réelle) ou par l'estimation de déplacements potentiels (connectivité potentielle). Les mesures de connectivité potentielle permettent de travailler sur de larges 
zones d'études avec une quantité de données moindre. Parmi les différentes méthodes existantes, la modélisation fondée sur les graphes paysagers permet d'évaluer la connectivité potentielle de manière pertinente (Urban et al., 2009). Fort de ce constat, l'évaluation de la connectivité écologique, réalisée dans le cas d'application présenté, s'appuie sur des graphes paysagers. Dans la suite de cet article, le terme général " connectivité écologique » est utilisé spécifiquement pour désigner la connectivité fonctionnelle potentielle des paysages.

À l'instar des travaux en écologie du paysage, la théorie des graphes offre des perspectives prometteuses en géomorphologie. Abstraire la structure d'un réseau sous forme de graphe, en ne conservant que les nœuds (i.e. ici les sources de sédiments, les réservoirs, l'exutoire) et les liens (i.e. ici la mise en relation par un agent de transport) est classique (fig. 3). En revanche, analyser à travers les outils de théorie algébrique de graphes la structure d'un graphe orienté, forcé par les lois de la gravité, représente un verrou scientifique à lever.

Deux démarches complémentaires issues de la théorie des graphes ont offert de premiers résultats pour évaluer la connectivité hydrosédimentaire. Une " analyse du flot » vise à affecter d'une valeur simulant la réalité à chaque arête (Heckmann et Schwanghart, 2013 ; Heckmann et al., 2015). Cette valeur correspond à la quantité de matière transportée le long de ce lien (i.e. flux de sédiments), de telle sorte qu'en chaque nœud la somme des valeurs des liens entrants soit égale à la somme des valeurs des liens sortants (loi de Kirchhoff). Il s'agit d'une simulation, dans la mesure où cette analyse se fonde sur une hypothèse de conservation des flux. Idéalement, il s'agirait toutefois d'affecter une valeur correspondant à l'aptitude de la source à fournir des sédiments, révélée par des observations de terrain. L'objectif est de voir comment l'agencement des liens dans le graphe, la structure du réseau, prédispose l'agencement et la valeur des flux sédimentaires (fig. 4A). Parfois qualifié d'effet réseau (Cossart, 2016 ; Cossart et Fressard, 2017), cette prédisposition du réseau à drainer et organiser les flux de sédiments toute chose égale par ailleurs peut être considérée comme sa contribution nette aux flux. La théorie des graphes aide alors à identifier des assemblages récurrents entre des réservoirs sédimentaires, ou des associations récurrentes entre des réservoirs sédimentaires et des processus qui contribuent à leur alimentation et/ou leur démantèlement (fig. 4B). Cette démarche permet également d'identifier des sous-systèmes qui ne sont pas reliés à l'exutoire, qui dirigent les sédiments vers des puits. Il s'agit de composantes connexes, c'est-à-dire de sous-graphes indépendants, mais au sein desquels les nœuds sont tous connectés l'un à l'autre. L'identification de ces composantes connexes aide à estimer la fragmentation spatiale de la cascade de sédiments : il s'agit d'estimer un sediment delivery ratio (i.e. volume de sédiments exportés à l'exutoire / volume de sédiments produits par les sources) et de révéler l'étendue de la zone qui peut potentiellement contribuer à l'exportation sédimentaire (Heckmann et Schwanghart, 2013 ; Heckmann et al., 2015 ; Cossart et Fressard, 2017 ; Heckmann et Vericat, 2018). Au final, il s'agit d'évaluer dans quelle mesure les sédiments se déplacent de manière davantage fragmentée que les flux liquides qui les transportent.

En complément de cette approche englobant l'ensemble du système que constitue le bassin-versant, la théorie des graphes offre également le cadre méthodologique pour développer des métriques qui permettent de hiérarchiser l'importance de points névralgiques au sein de la cascade sédimentaire (fig. 4). Mesurer la connectivité dans un réseau inscrit dans l'espace, et donc l'aptitude des nœuds à être reliés entre eux par les liens du réseau, mobilise la notion de distance. Il s'agit, pour chaque nœud, de mesurer son accessibilité depuis tous les autres points du réseau, ou depuis tous les nœuds
A

B

C
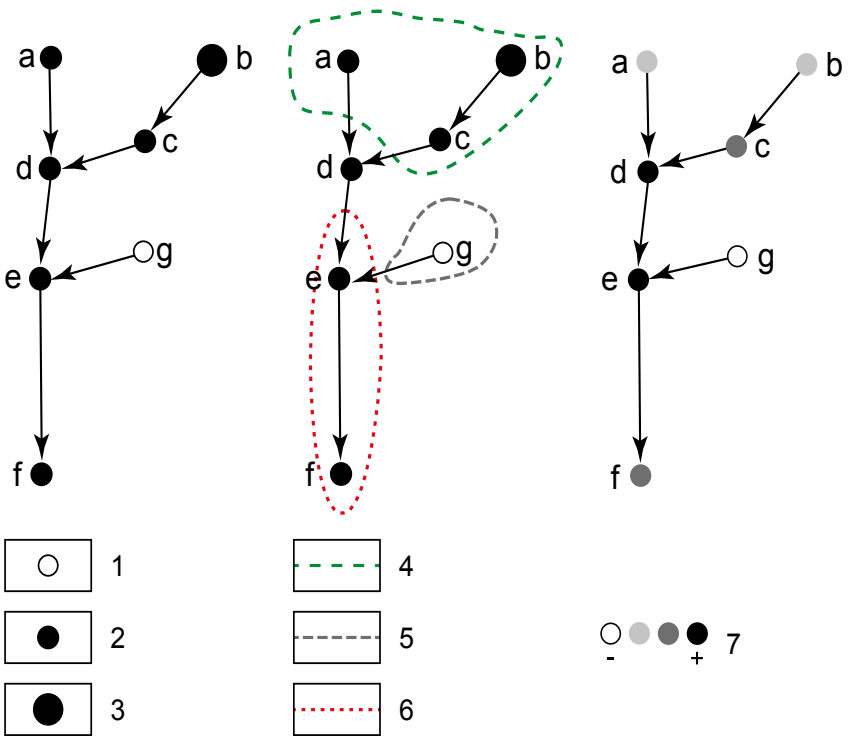

$+\quad+7$

Fig. 4 - Estimation de la connectivité sédimentaire à partir de graphes.

A : Le graphe représente la fourniture sédimentaire à l'état initial du système et de la simulation ; B : Le calcul de la connectivité en un nœud (d) dépend d'une composante amont (qui contribue) à l'alimentation), et d'une composante avale, regroupant tous les nœuds dont le comportement est directement influencé par (d). C : Exemple d'agencement spatial de la connectivité à l'échelle du bassin-versant, permettant d'identifier les points névralgiques (d) et (e). 1. Absence de fourniture sédimentaire ; 2. Fourniture moyenne; 3. Fourniture importante; 4 . Composante amont : fourniture sédimentaire potentielle de $(\mathrm{d}) ; 5$. Pas d'influence directe sur $d ; 6$. Composante aval : chaîne de réservoirs alimentés par (d) ; 7. Indice de connectivité hiérarchisé.

Fig 4 - Assessment of sediment connectivity through graph theory.

A: Graph exhibits sediment supply (initial conditions of the system). B: The connectivity index is assessed in $(d)$, depends on the main characteristics of upslope and downslope components which encompass all the nodes directly fed by (d); C: Spatial pattern of sediment connectivity at catchment scale allowing the identification of hotspots $(d)$ and (e). 1. No sediment yield; 2. Medium sediment yield; 3. Large sediment yield; 4. Upslope component: potential sediment supply on (d); 5. No direct influence on (d); 6. Downslope component: reservoirs supplied by $(d)$; 7 . Hierarchical connectivity index.

situés en amont et/ou en aval en cas de flux forcés par la gravité (comme dans le cas des cascades hydro-sédimentaires). Positionner chaque nœud relativement aux autres permet ainsi de caractériser sa centralité et donc son aptitude à intercepter structurellement (de par sa situation stratégique) une grande part du flux qui anime le réseau (Cossart et Fressard, 2017). Cependant, mesurer une distance, un éloignement, nécessite de faire des choix méthodologiques. Par exemple, dans le cas d'une cascade sédimentaire, les flux sont facilités ou entravés en fonction de l'état de surface et des obstacles éventuels. Une typologie des obstacles est déjà bien établie et admise dans la communauté des géomorphologues, i.e. "barriers", «buffers ", «blankets" (Fryirs et al., 2007). À l'instar des travaux menés en écologie du paysage, la distance prise en compte dans ces réseaux ne doit alors pas simplement mesurer un éloignement kilométrique, mais plutôt une distance-coût, reflétant la difficulté $\mathrm{du}$ parcours. Pour estimer une telle distance, l'éloignement géométrique est alors associé à la rugosité de l'état de surface, ou encore au gradient de pente. Il a ainsi été remarqué qu'un nœud qui minimise la distance à plusieurs sources sédimentaires ainsi que la distance à l'exutoire correspond à un point névralgique du système, où peuvent intervenir des phénomènes de blocage puis de purge sédimentaire, en fonction d'une concurrence entre les flots entrant. Ce nœud influence directement le signal sédimentaire en sortie. 


\section{Cas d'application : mesurer la connectivité pour évaluer les impacts écologiques des formes d'urbanisation}

\subsection{Exemples de cas d'application}

Dans le champ de l'écologie du paysage, plusieurs travaux de recherche menés généralement au cours des dix dernières années s'appuient sur les graphes paysagers pour mesurer la perte de connectivité causée par les modifications de la matrice paysagère. Certains travaux privilégient une approche rétrospective en évaluant les pertes de connectivité imputables à la construction de grandes infrastructures de transports (Fu et al., 2010 ; Girardet et al., 2013 ; Clauzel et al., 2013) ou plus généralement aux changements de l'occupation du sol (Sahraoui et al., 2017). À partir de métriques de connectivité fonctionnelle calculées à l'aide de graphes paysagers, plusieurs travaux de recherche identifient des zones prioritaires pour la conservation de certaines espèces et le maintien de la connectivité des réseaux écologiques (Zetterberg et al., 2010 ; García-Feced et al., 2011 ; Gurrutxaga et al., 2011 ; Foltête et al., 2014). D’autres travaux se basent sur une approche prospective en évaluant l'impact potentiel de différents scénarios d'aménagement du territoire tels que la construction d'une nouvelle autoroute (Vasas et al., 2009) ou de nouvelles zones de développement résidentiel (Tannier et al., 2012 ; Bourgeois, 2015 ; Tannier et al., 2016a).
4.2. Évaluation des impacts écologiques des formes d'urbanisation

L'objectif principal de la démarche présentée dans cette soussection vise à évaluer l'impact écologique de différentes formes de croissance urbaine à l'aide d'une démarche prospective, entre 2010 et 2030, dans une zone de $60 \mathrm{~km}$ englobant l'aire urbaine de Besançon (Doubs) (fig. 5). Les étapes du travail sont les suivantes : (i) simulation de scénarios d'urbanisation, (ii) modélisation des graphes paysagers de plusieurs espèces à l'état initial et pour chaque scénario et (iii) estimation de la perte de connectivité imputable à chaque scénario en calculant le taux de variation d'une métrique paysagère entre l'état initial et chaque scénario d'urbanisation.

\subsubsection{Simulation des évolutions urbaines}

Cinq scénarios sont simulés : ville compacte, ville modérément compacte, périurbain régulé, étalement urbain et Transit Oriented Development (Bourgeois, 2015 et Tannier et al., 2016a pour plus de détails). Les formes urbaines de chacun d'entre eux sont volontairement contrastées mais le nombre de logements construits reste le même pour chaque simulation. Pour chacun de ces scénarios, le trafic routier est simulé à l'aide d'une plateforme de simulation multi-agents (Tannier et al., 2016b). Ainsi, le nombre de véhicules par jour est estimé à l'état initial de la simulation, puis pour chacun des scénarios et ce, pour chaque tronçon routier de la zone d'étude.

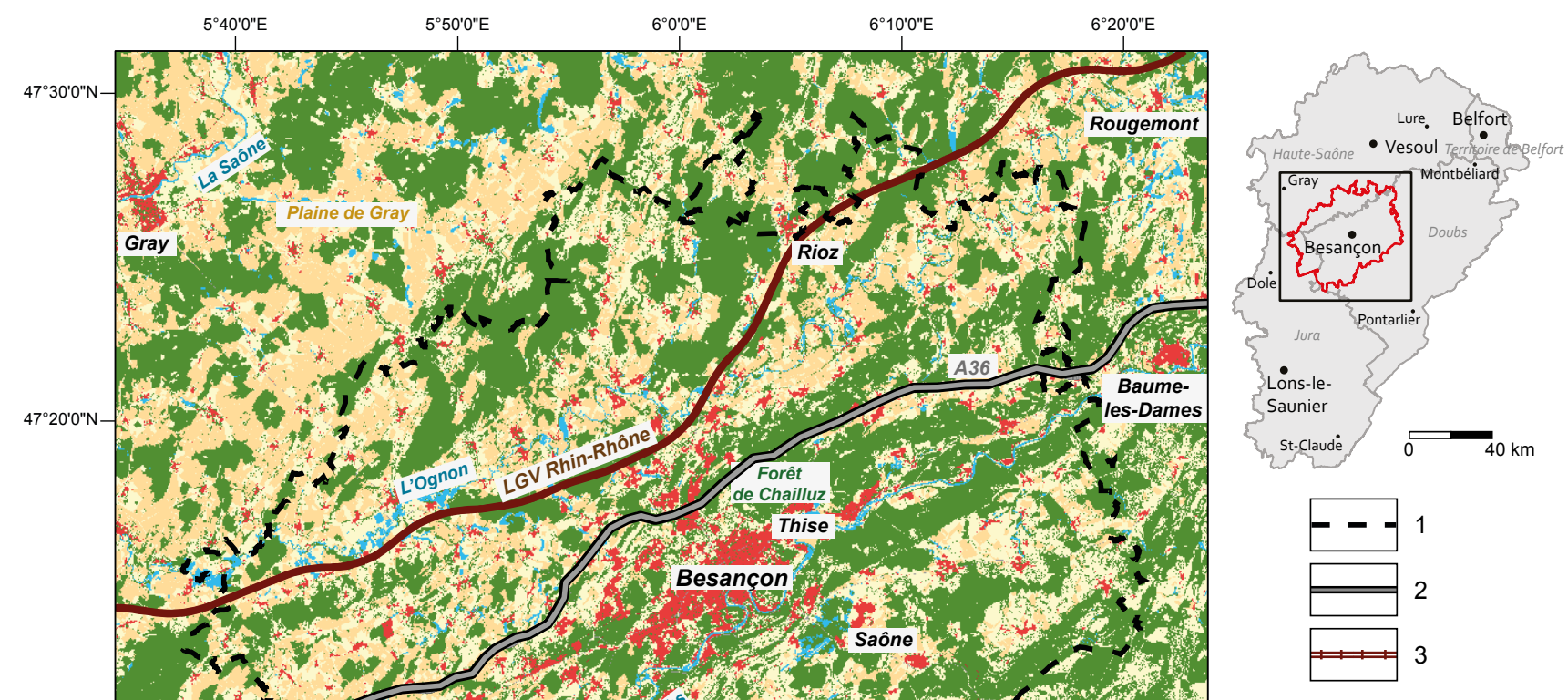

Classes d'occupation du sol

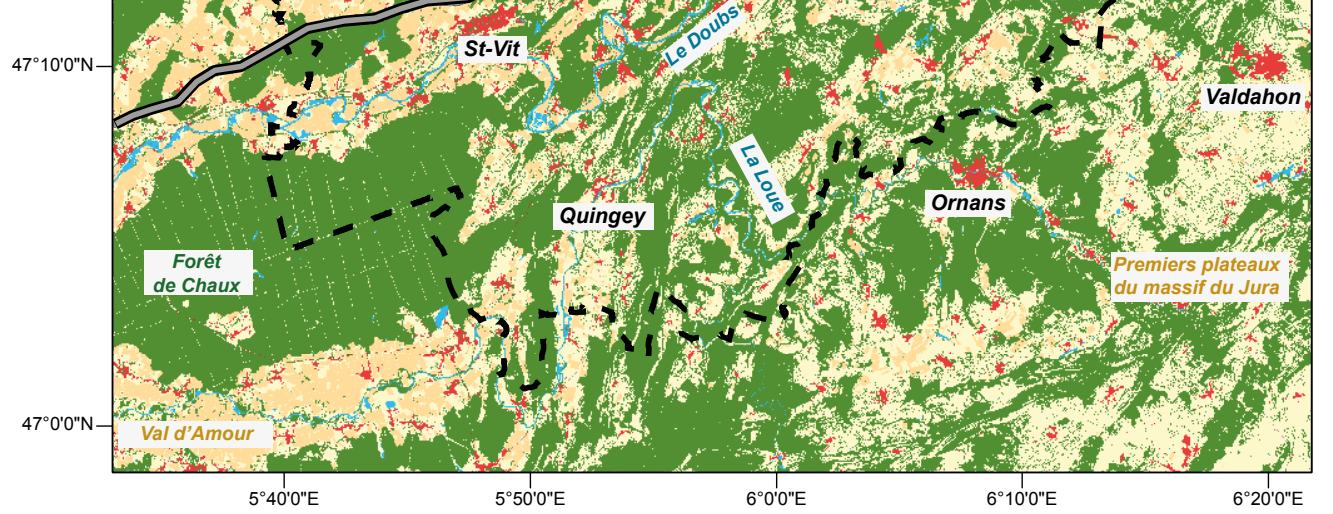

Land use classes

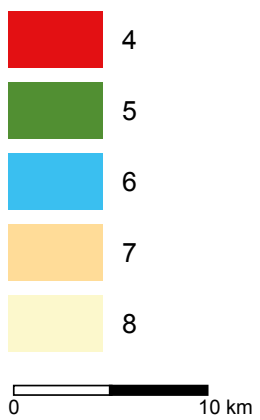

Fig. 5 - Zone d'étude : l'aire urbaine de Besançon.

1. Aire urbaine de Besançon ; 2. Autoroute ; 3 . LGV ; 4. Zone urbanisée ; 5. Forêt; 6. Surface en eau et zone humide; 7 . Culture; 8 . Prairie et sol nu.
Fig 5 - Study area: urban area of Besançon.

1. Urban area of Besançon; 2. Highway; 3. High-speed railway; 4. Urban area; 5. Forest; 6. Water and wetland; 7. Crop; 8. Meadow and bare ground. 


\subsubsection{Modélisation des réseaux écologiques}

Pour une espèce donnée, l'impact d'un scénario d'urbanisation est estimé en mesurant la perte de connectivité de son habitat entre l'état initial de l'occupation du sol et l'état final suite au processus d'urbanisation simulée. Toutefois, compte-tenu de la variété des besoins des différentes espèces animales, travailler sur une seule espèce cible serait trop réducteur pour caractériser l'impact écologique de chaque scénario d'urbanisation (Lindenmayer et al., 2000). En effet, un même paysage peut présenter un degré de connectivité élevé pour certaines espèces, et plus faible pour d'autres (Taylor et al., 1993 ; Beier et Noss, 1998 ; Bennett, 1999 ; Hess et Fischer, 2001). Chaque espèce est dépendante de la configuration et de la composition de la mosaïque paysagère pour trois raisons principales : (i) chaque espèce possède un biotope qui lui est propre, (ii) chaque espèce a des exigences différentes en termes de surface du domaine vital et (iii) chaque espèce dispose de ses propres capacités de mouvement qui sont liées à la distance entre ses taches d'habitat et la nature des éléments de la matrice à traverser (Vos et al, 2001; Opdam et al., 2002). Cependant, en pratique, il n'est pas possible de prendre en compte les contraintes et les exigences de toutes les espèces présentes dans la zone d'étude par rapport à la mosaïque paysagère.

L'approche multi-espèces, consistant à prendre comme référence une série d'espèces cibles, nous paraît être un bon compromis entre la modélisation et la réalité écologique. Pour cela, nous utilisons une approche par profil écologique, consistant à sélectionner une large gamme d'espèces susceptibles de représenter au mieux leur milieu et l'ensemble des processus au sein d'un paysage (Vos et al., 2001). La première étape de la démarche consiste à obtenir un nombre réduit d'espèces après l'application de plusieurs filtres : présence dans la zone d'étude, espèces déterminantes Trame Verte et Bleue (Sordello et al., 2011), espèces potentiellement menacées par l'urbanisation, données suffisamment disponibles et possibilité de cartographie de leur habitat dans un SIG. Ces espèces sont ensuite regroupées à partir de trois critères proposés par Opdam et al. (2008) : l'écosystème dans lequel vit l'espèce, sa distance de dispersion et la taille minimale de ses taches d'habitat. Au final, seize groupes d'espèces sont ainsi identifiés (par exemple les oiseaux de milieux ouverts grands disperseurs). Pour chacun de ces groupes, une espèce représentative est sélectionnée. Par exemple, le Chat forestier (Felis silvestris) représente les mammifères forestiers grands et moyens disperseurs.

Pour l'année 2010, une carte d'occupation du sol est réalisée pour chaque groupe d'espèces. Ces cartes sont construites au format matriciel à 10 mètres de résolution et comprennent 5 à 14 classes selon les groupes d'espèces. En effet, pour certains groupes, des classes d'occupation du sol spécifiques doivent être ajoutées comme les ruisseaux, les haies ou les lisières forestières qui peuvent dans certains cas constituer l'habitat de certaines espèces. Ces cartes sont distinguées en deux catégories : l'habitat des espèces (par exemple les zones forestières de plus de $1 \mathrm{ha}$ ) et la matrice paysagère, constituée de zones plus ou moins favorables aux déplacements entre les taches d'habitat. Pour chacune des classes d'occupation du sol, une valeur de coût est attribuée et s'échelonne entre 1 (habitat) et 10000 (barrière). Ces valeurs de coûts sont estimées en fonction des exigences écologiques de chaque groupe d'espèces d'après les informations recueillies dans la littérature scientifique. D’un point de vue spatial, la simulation d'un nouveau scénario se traduit par une modification de la carte d'occupation du sol initiale (fig. 6A), par ajout de nouvelles cellules de type «zones bâties » de 20 mètres de côté. L'augmentation ou la diminution du trafic simulé modifie
A

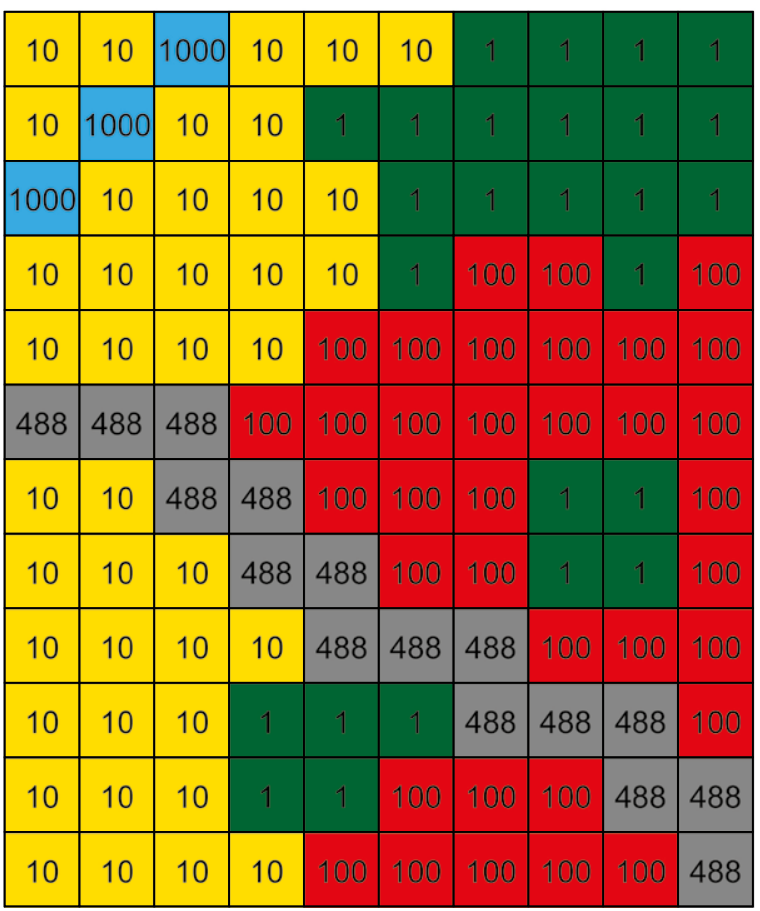

B

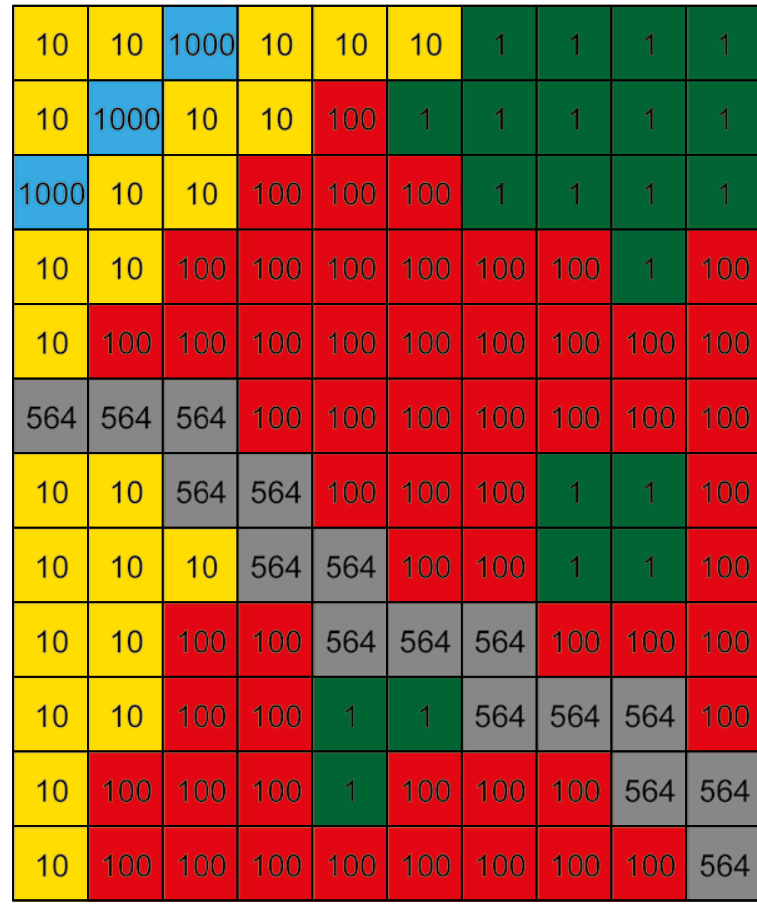

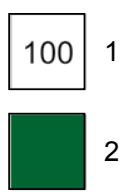
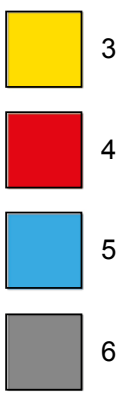

Fig. 6 - Exemple virtuel des changements de valeurs de coûts imputables à la construction de nouveaux espaces bâtis et à laugmentation du trafic entre létat initial et létat final.

D’après Bourgeois (2015). A : État initial ; B : Après simulation du développement résidentiel. 1. Coût attribué à chaque classe d'occupation du sol ; 2. Forêt (habitat); 3. Prairie; 4. Bâti; 5. Eau;6. Route.
Fig 6 - Virtual example of changing cost values resulting from newly built areas and traffic growth between initial state and final state.

After Bourgeois (2015). A: Initial state; B: After simulation of residential development and traffic. 1. Cost allocated to each land use class; 2. Forest (habitat); 3. Meadow; 4. Built area; 5 . Water; 6 . Road. 
également les coûts attribués aux cellules de type " route » entre l'état initial et l'état final, après chaque scénario d'urbanisation (fig. 6B).

Chaque carte d'occupation du sol constitue le support de modélisation d'un graphe paysager. Les nœuds du graphe sont constitués par les taches d'habitat et les liens du graphe sont représentés par les déplacements potentiels entre les taches d'habitat. Les valeurs de coûts attribuées à chaque classe d'occupation du sol permettent d'identifier les chemins de moindre coût entre les taches d'habitat (Adriaensen et al., 2003). Afin de prendre en compte la résistance de la matrice paysagère, la distance de dispersion, exprimée dans la littérature en unités métriques doit être convertie en unité de coût. Chaque lien du graphe est alors caractérisé par la distance métrique entre les deux taches qu'il relie, et par la distance de coûts cumulés entre ces deux mêmes taches. Celle-ci est utilisée pour réaliser le seuillage du graphe. Elle est spécifique à chaque groupe d'espèces et à sa carte d'occupation du sol associée. En cas de modification des valeurs de coûts, cette valeur doit être recalculée. Les déplacements potentiels modélisés dans les graphes paysagers correspondent ainsi aux mouvements potentiels de dispersion des individus entre leurs taches d'habitat, représentés en unités de coûts. Un graphe paysager est ainsi calculé, pour chaque groupe d'espèces, à l'état initial et pour chaque scénario d'urbanisation à l'aide du logiciel Graphab (Foltête et al., 2012).

\subsubsection{Calcul des métriques de connectivité}

Afin d'évaluer la perte de connectivité imputable à chaque scénario d'urbanisation, il est nécessaire de calculer une métrique de connectivité répondant à certains critères importants dans le cadre de cette application : (i) elle doit prendre en compte à la fois l'étendue des surfaces d'habitat (potentiel démographique) et leur degré de connectivité (potentiel de mouvement) ; (ii) sa valeur doit être normalisée, afin de pouvoir établir des comparaisons entre les différents graphes paysagers créés pour chaque scénario d'urbanisation ; (iii) sa valeur doit être plus faible en cas de diminution de la surface des taches d'habitat par exemple si de nouveaux espaces bâtis y sont construits ; (iv) elle doit prendre en compte la modification éventuelle des chemins de moindre coût entre les taches si les valeurs de coût attribuées à la matrice paysagère sont changées suite aux variations du trafic ou à la construction de nouveaux espaces bâtis. Elle doit donc considérer l'augmentation potentielle de la distance entre les taches d'habitat ; $(v)$ elle doit considérer la perte de toutes les taches d'habitat, qu'elles soient connectées ou non connectées. À ce titre, la valeur de la métrique doit également diminuer plus fortement si une tache ayant une fonction de pas japonais (stepping-stone) clé est supprimée.

L'utilisation de la métrique $P C$ permet de répondre de manière satisfaisante à l'ensemble de ces critères (Saura et Pascual-Hortal, 2007). La métrique $P C$ permet ainsi de caractériser la probabilité que des individus placés au hasard dans la zone d'étude se situent dans des taches d'habitat connexes. Elle intègre la probabilité de dispersion directe $p$ en fonction de la distance entre les taches d'habitat :

$$
P C=\frac{\sum_{i=1}^{n} \sum_{j=1}^{n} a_{i} a_{j} p_{i j}^{*}}{A_{L}^{2}}
$$

où $a_{i}$ et $a_{j}$ sont les surfaces respectives des taches $i$ et $j, p_{i j}^{*}$ le produit maximum des probabilités de dispersion de tous les parcours possibles entre la tache $i$ et $j$ et $A_{L}$ la surface totale de la zone d'étude. En étant normalisé par la surface de la zone, l'indice $P C$ s'interprète comme une probabilité. Ses valeurs sont donc comprises entre 0 et 1.
S'il n'existe pas d'habitat dans la zone d'étude, $P C=0$ puisque $\alpha=0$. S'il n'y a qu'une seule tache dans la zone d'étude avec $a_{i}=A_{L}$, donc $a_{i}=a_{j}$ par conséquent $p_{i j}=1$ et $\mathrm{PC}=1$.

Le $P C$ est donc la métrique globale retenue dans ce travail pour mesurer la connectivité de l'ensemble des réseaux écologiques modélisés. Le $P C$ présente l'avantage de prendre en compte la pondération des liens en fonction de la distance séparant les taches d'habitat. La distance maximale de dispersion (en unités de coûts) est utilisée de manière indicative pour déterminer le facteur $a$ nécessaire au calcul de la probabilité de dispersion $p_{i j}$ de l'espèce considérée. La métrique est calculée pour l'ensemble du réseau mais ne permet pas de savoir quels éléments du graphe présentent le plus fort (ou le plus faible) degré de connectivité. Pour cela, des métriques locales de connectivité peuvent être utilisées. Le $P C_{f l u x}$ caractérise la contribution de chaque tache à l'indice $P C$ global. L'utilisation de cette métrique permet de comparer sa valeur avant et après simulation d'un scénario d'urbanisation. Pour une tache donnée $j$, $P C_{f l u x}(j)$ correspond à :

$$
P C_{\text {flux }}(j)=\frac{\sum_{i=1}^{n} a_{i} a_{j} p_{i j}^{*}}{A_{L}^{2}}
$$

où $a_{i}$ et $a_{j}$ sont les surfaces respectives des taches $i$ et $j, p_{i j}^{*}$ le produit maximum des probabilités de dispersion de tous les parcours possibles entre la tache $i$ et $j$ et $A_{L}$ la surface totale de la zone d'étude. Ainsi, la somme de toutes les valeurs de $P C_{f l u x}$ de chaque tache est égale à la valeur globale du $P C$ calculée pour le même graphe.

\subsubsection{Exemples de résultats}

La construction de graphes paysagers pour chaque groupe d'espèces et le calcul de l'indice $P C$ associé à chacun d'entre eux a permis d'évaluer l'impact écologique des formes d'urbanisation pour l'ensemble de la zone d'étude. Ces impacts globaux ont été calculés pour chaque scénario et chaque groupe. Deux aspects principaux ressortent de ces résultats : les espèces liées aux milieux ouverts sont plus sensibles à l'urbanisation, principalement car ces espaces sont plus urbanisés que les zones boisées. De plus, pour la majeure partie des espèces étudiées, la ville compacte est le modèle de ville qui limite le plus la perte de connectivité (fig. 7). Ces résultats montrent également que l'étalement urbain est la forme de ville responsable des impacts écologiques les plus importants.

À partir de ces résultats, nous avons également pu montrer que le facteur permettant la différenciation des impacts des scénarios pour un groupe d'espèces donné est le développement résidentiel en lui-même, plus que le trafic simulé. Toutefois, pour comparer les impacts de groupes d'espèces entre eux, la prise en compte du trafic est importante.

Plusieurs facteurs permettent d'expliquer que les variations du $P C$ soient globalement peu importantes : la faible quantité d'urbanisation simulée, le réalisme des scénarios, et la grande taille de la zone d'étude où les évolutions urbaines ne sont simulées que sur $44 \%$ du territoire. Cependant, il faut être conscient que ces valeurs représentent des pertes de connectivité potentielle. Il est possible qu'une diminution du PC, même très faible dans le modèle ait des conséquences écologiques importantes dans la réalité. Les valeurs obtenues servent donc plus à hiérarchiser les scénarios et les espèces entre elles qu'à évaluer un impact écologique « absolu ».

Au niveau local, l'impact écologique de chaque scénario a été estimé pour chaque tache d'habitat pour tous les groupes d'espèces retenus (fig. 8). La distribution spatiale des impacts n'est pas aussi simple qu'elle n'y parait puisque les réseaux écologiques de certaines espèces peuvent être modifiés dans des zones qui ne sont pas 


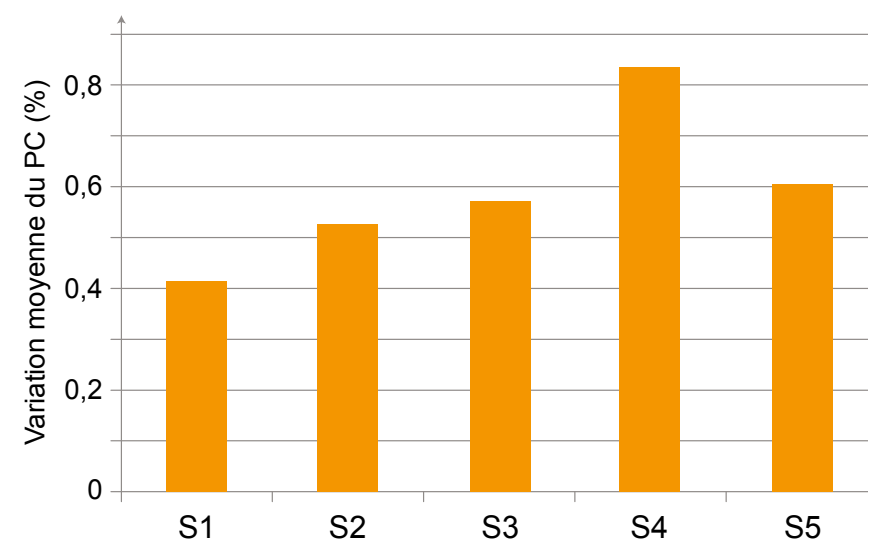

Fig. 7 - Impact écologique moyen de chaque scénario d'urbanisation sur les seize groupes d'espèces sélectionnés.

D'après Bourgeois (2015). Taux de variation moyen du PC normalisé entre 0 et 1 pour chaque groupe d'espèces et scénarios de développement résidentiel : S1. Ville compacte ; S2. Ville modérément compacte ; S3. Périurbain régulé ; S4. Étalement urbain ; S5. Transit Oriented Development.

Fig 7 - Average ecological impact of each urbanization scenario on sixteen selected species groups.

After Bourgeois (2015). Average variation rate of PC normalized between 0 and 1 for each species group and scenarios of residential development: S1. Compact residential development; S2. Moderately compact development; S3. Controlled development of suburb areas; S4. Urban sprawl; S5. Transit Oriented Development. forcément adjacentes aux nouvelles évolutions urbaines. Pour cette raison, il est difficile de savoir exactement à quelle évolution urbaine est liée la perte de connectivité d'une tache d'habitat donnée.

\section{Cas d'application : mesurer la connectivité pour évaluer les impacts géomorphologiques de la déglaciation}

\subsection{Le bassin-versant de Celse-Nière}

Nous avons sélectionné une étude de cas menée dans les Alpes françaises du Sud (bassin versant Celse-Nière, massif des Ecrins), soit un bassin-versant où les perturbations anthropiques sur la cascade sédimentaire sont absentes (absence de barrage et de toute structure hydraulique, absence d'activité agricole, etc.). Ce bassin versant constitue donc un laboratoire à ciel ouvert permettant d'étudier la variabilité des signaux sédimentaires dans un contexte de changement climatique : le bassin-versant subit une tendance notable au retrait glaciaire depuis le Petit Âge de Glace (fig. 9). L'englacement actuel de ce bassin-versant est restreint (environ $5 \mathrm{~km}^{2}$ ), malgré les hautes altitudes (L'Ailefroide, point culminant, atteint $3954 \mathrm{~m}$ ). La zone d'étude est en effet en partie abritée des influences océaniques d'ouest par la ligne de crêtes faitière du massif des Écrins ; par exemple, les précipitations sont d'environ $995 \mathrm{~mm} /$
A

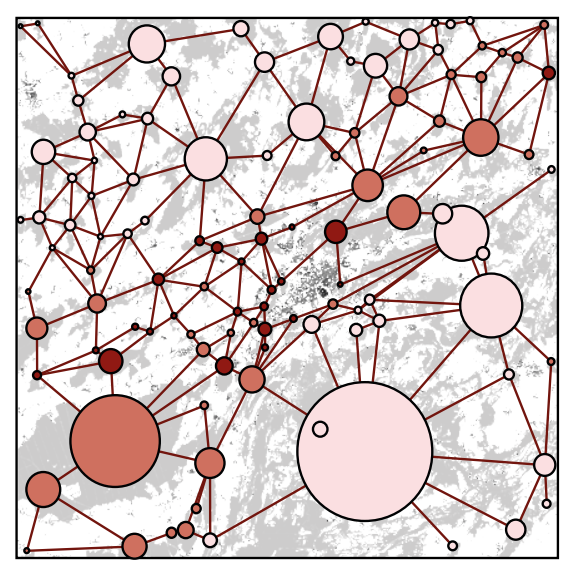

$\mathrm{D}$

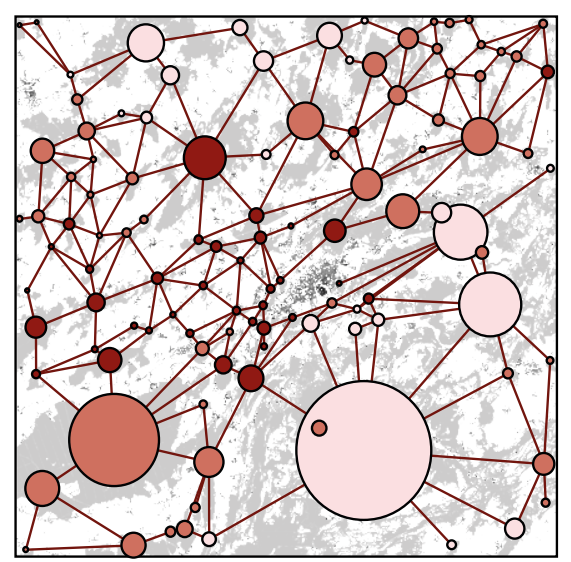

$\mathrm{B}$

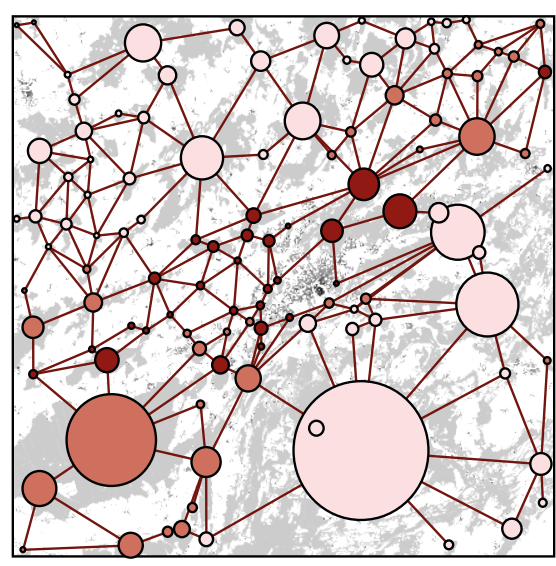

$\mathrm{E}$

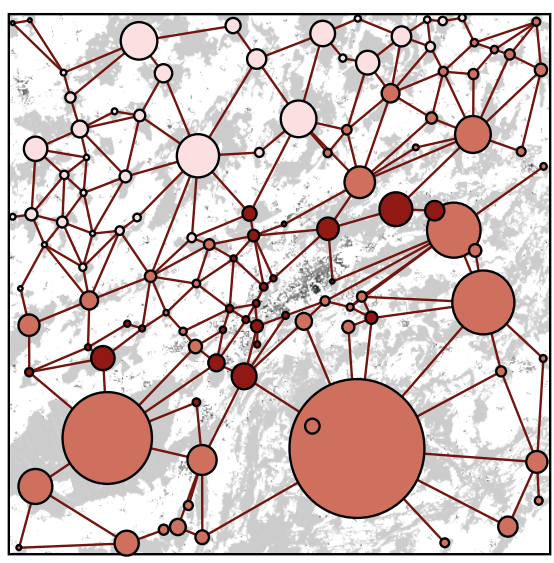

C
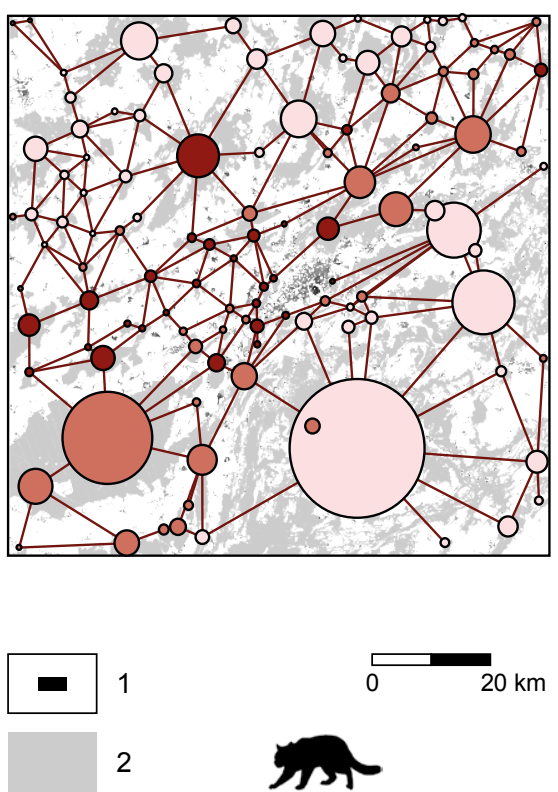

Graphe paysager

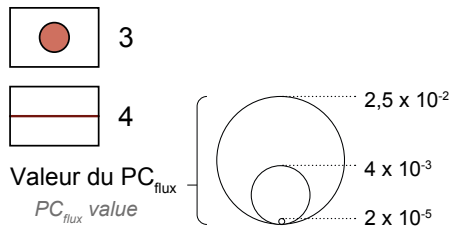

Taux de variation du $\mathrm{PC}_{\text {flux }}(\%)-$

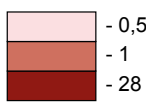

Variation rate of $P C_{f u x}(\%)$

Fig. 8 - Changements de la connectivité écologique de chaque tache d'habitat du groupe "Chat forestier " pour chaque scénario simulé.

D’après Bourgeois (2015). A : Ville compacte ; B : Ville modérément compacte ; C : Périurbain régulé ; D : Étalement urbain; E : Transit Oriented Development. 1. Bâti initial; 2. Forêt (habitat) ; 3. Nœud ; 4. Lien.
Fig 8 - Variations of ecological connectivity for each habitat patch of "Wildcat" group for each simulated scenario.

After Bourgeois (2015). A: Compact residential development; B: Moderately compact development; C: Controlled development of suburb areas; D: Urban sprawl; E: Transit Oriented Development. 1. Initial buildings; 2. Forest (habitat); 3. Node; 4. Link. 
an à la station de Pelvoux (1 $280 \mathrm{~m}$ d'altitude) à proximité du terrain étudié, un niveau nettement inférieur à celui observé sur le flanc ouest du Massif des Écrins (1 $195 \mathrm{~mm} / \mathrm{an}$ à la station de Valjouffrey, $1160 \mathrm{~m})$. L'altitude de la ligne d'équilibre est ainsi évaluée entre
3100 et $3300 \mathrm{~m}$ (Cossart, 2013), soit $200 \mathrm{~m}$ plus haut que dans la partie ouest du Massif des Écrins.

$\mathrm{Au}$ cours du Petit Âge de Glace (PAG), une langue glaciaire principale (glacier du Sélé) occupait la vallée; son front était situé

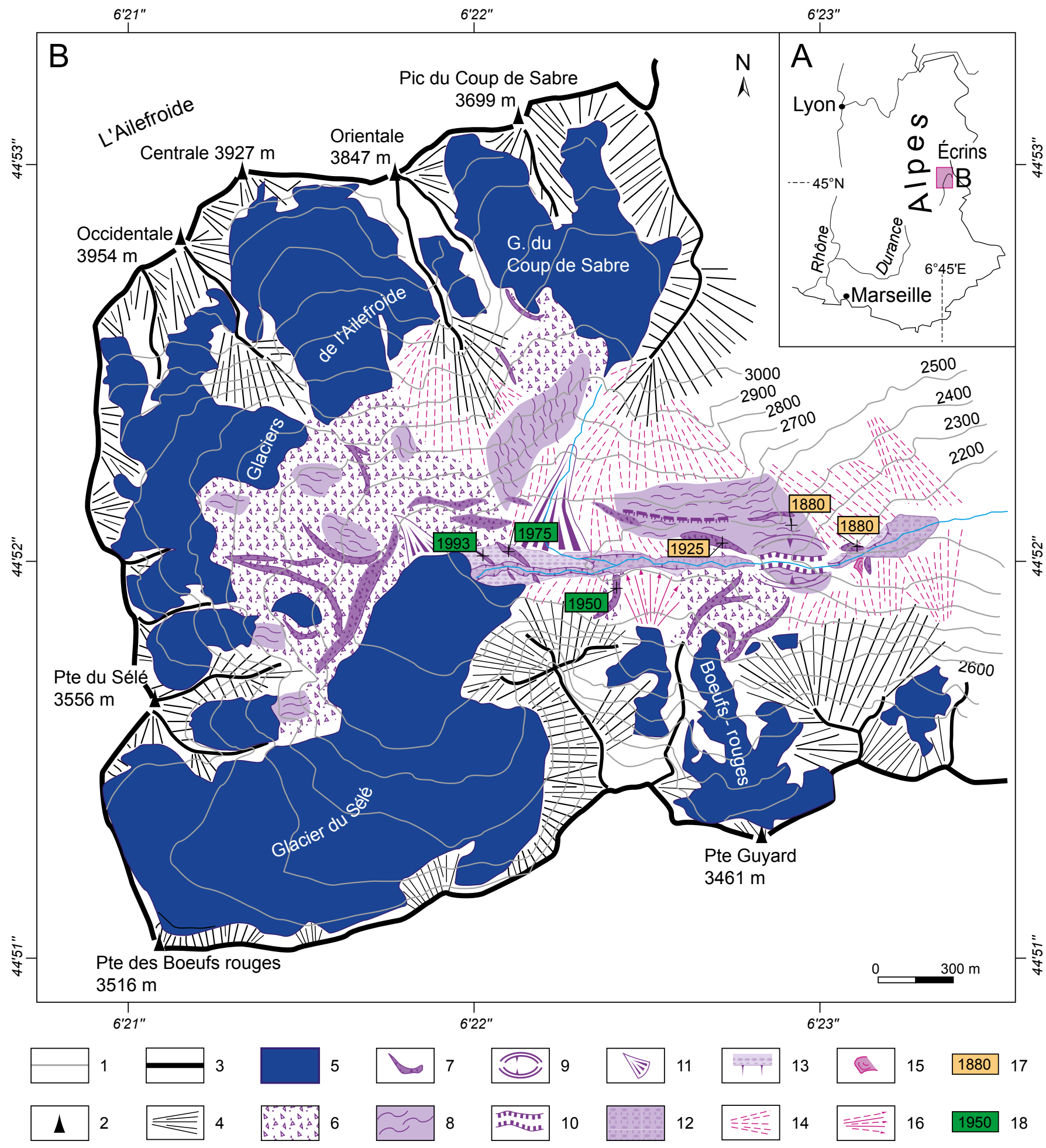

Fig. 9 - Croquis géomorphologique du bassin-versant de Celse-Nière (d'après Cossart et Fort, 2008).

A : Localisation du secteur d'étude ; B : Croquis géomorphologique. 1. Courbe de niveau ; 2. Sommet ; 3. Ligne de crête ; 4. Paroi raide ; 5 . Glacier (été 2002) ; 6. Till ; 7. Moraine latéro-frontale ; 8 . Roche Moutonnée ; 9 . Verrou ; 10 . Gorge sous glaciaire ; 11. Cône pro-glaciaire; 12 . Alluvions fluvio-glaciaires; 13 . Terrasse fluvio-glaciaire; 14. Éboulis ; 15. Glacier rocheux ; 16. Cône mixte (avalanche, éboulis); 17. Datation par lichénométrie ; 18. Datation par photographie aérienne.
Fig. 9 - Geomorphological sketch of Celse-Nière catchment (after Cossart and Fort, 2008).

A: Location of the study area; B: Geomorphological sketch. 1. Contour line; 2. Summit; 3. Main ridges; 4. Steep slope; 5. Glacier (summer 2002); 6. Till; 7. Morainic ridges; 8. Roches Moutonnées; 9. Glacial knob; 10. Subglacial gorge; 11. Proglacial cone; 12. Glacio-fluvial deposits; 13. Glacio-fluvial terrace; 14. Scree cone; 15. Rock glacier 16. Avalanches and scree deposits; 17. Lichenometric dating; 18. Aerial photographs dating. 
à $2250 \mathrm{~m}$ Dans la partie supérieure du bassin versant, des affluents glaciaires (Ailefroide, Coup-de-Sabre) convergeaient avec la langue du glacier de vallée. La superficie totale couverte par les glaciers était alors d'environ $9 \mathrm{~km}^{2}$. En 2013, seul le glacier du Sélé atteint encore le fond de la vallée. Les glaciers affluents se sont retranchés à plus de $3000 \mathrm{~m}$ d'altitude sous forme de glaciers suspendus, qui alimentent en sédiments et en eaux de fonte des cascades sédimentaires élémentaires jusqu'au fond de vallée (fig. 9). La cascade de sédiments a déjà été conceptualisée (fig. 10) et une approche quantitative a estimé que l'ensemble de ce secteur avait exporté environ $60.10^{3} \mathrm{~m}^{3}$ depuis le PAG (Cossart et Fort, 2008 ; Cossart, 2016).

\subsection{Formalisation des données}

Fondée sur une expertise géomorphologique, une carte géomorphologique a été dressée dans un logiciel SIG (QGIS). Les observations ont été réalisées sur le terrain (en 2002, 2003, 2006, 2012) et ont été complétées par l'examen des photographies aériennes (publiées par l'IGN). Les photographies acquises lors des années 1952 et 1981 ont été étudiées en raison de la très faible couverture nivale. À l'échelle locale, une attention particulière a été portée aux objets géomorphologiques susceptibles de créer des obstacles aux flux de sédiments, en reprenant la typologie de Fryirs et al. (2007) : "barriers », " buffers ", " blankets ». Premièrement, dans un tel contexte paraglaciaire, les crêtes morainiques (latérales et frontales) créent un niveau de base local qui interrompt les flux sédimentaires et jouent le rôle de barrière (Cossart et Fort, 2008). Deuxièmement, les terrasses fluvio-glaciaires déposées sur les parties externes du fond de vallée constituent une zone tampon qui déconnecte les versants du talweg. De même, les roches-moutonnées peuvent également constituer des zones tampons (Cossart, 2016). Troisièmement, dans ce type de contexte alpin, les cours d'eau ne peuvent transporter les sédiments à granulométrie trop grossière apportés par les dynamiques gravitaires de versant (Caine, 1984).
Ainsi, dans la plupart des cas, le matériel sédimentaire transporté vers le fond de vallée par des glissements de terrain, des coulées de débris, l'éboulisation dépassent la capacité de transport des cours d'eau et ne peuvent alimenter efficacement la cascade sédimentaire (Rapp, 1960 ; Jackli, 1957).

Afin de décrire quantitativement les assemblages de formes et de liens constituant la cascade sédimentaire, nous la représentons sous la forme d'un graphe orienté suivant une procédure décrite par Cossart et Fressard (2017). Un inventaire exhaustif des liens est fourni à partir d'un réseau de nœuds réguliers (la distance entre nœuds est égale à $100 \mathrm{~m}$ ). Les nœuds correspondent à des unités géomorphologiques (sources sédimentaires, zones de stockage ou puits sédimentaires) et sont caractérisés par le type de formation superficielle qui les constitue. Deux nœuds peuvent être reliés par des liens, qui se réfèrent au cheminement sédimentaire. Si deux nœuds $i$ et $j$ sont considérés comme joints ou adjacents dans la cascade sédimentaire par l'expertise géomorphologique, alors un lien de $i$ à $j$ est formalisé dans le SIG. A contrario si une barrière, une zone tampon ou tout phénomène d'entrave sédimentaire est observé, aucun lien n'est formalisé entre les nœuds. La grille dessinée par les nœuds est volontairement régulière afin de constituer un canevas stable dans le temps, facilitant les comparaisons diachroniques dans l'agencement de la cascade sédimentaire. Des changements de propriétés de chaque nœud (e.g. nature des formations superficielles) ou encore des changements dans l'agencement des liens peuvent ainsi être identifiés entre deux dates.

\subsection{Calcul de métriques : la connectivité structurelle}

Comme dans les graphes non orientés, un premier objectif consiste à quantifier «l'effet réseau » (Cossart et Fressard, 2017) pour mettre en évidence l'effet de la structure spatiale du réseau sur la quantité de sédiments exportés à l'exutoire. Sous l'hypothèse de toutes choses étant égales par ailleurs, un volume virtuel de sédiments (1)

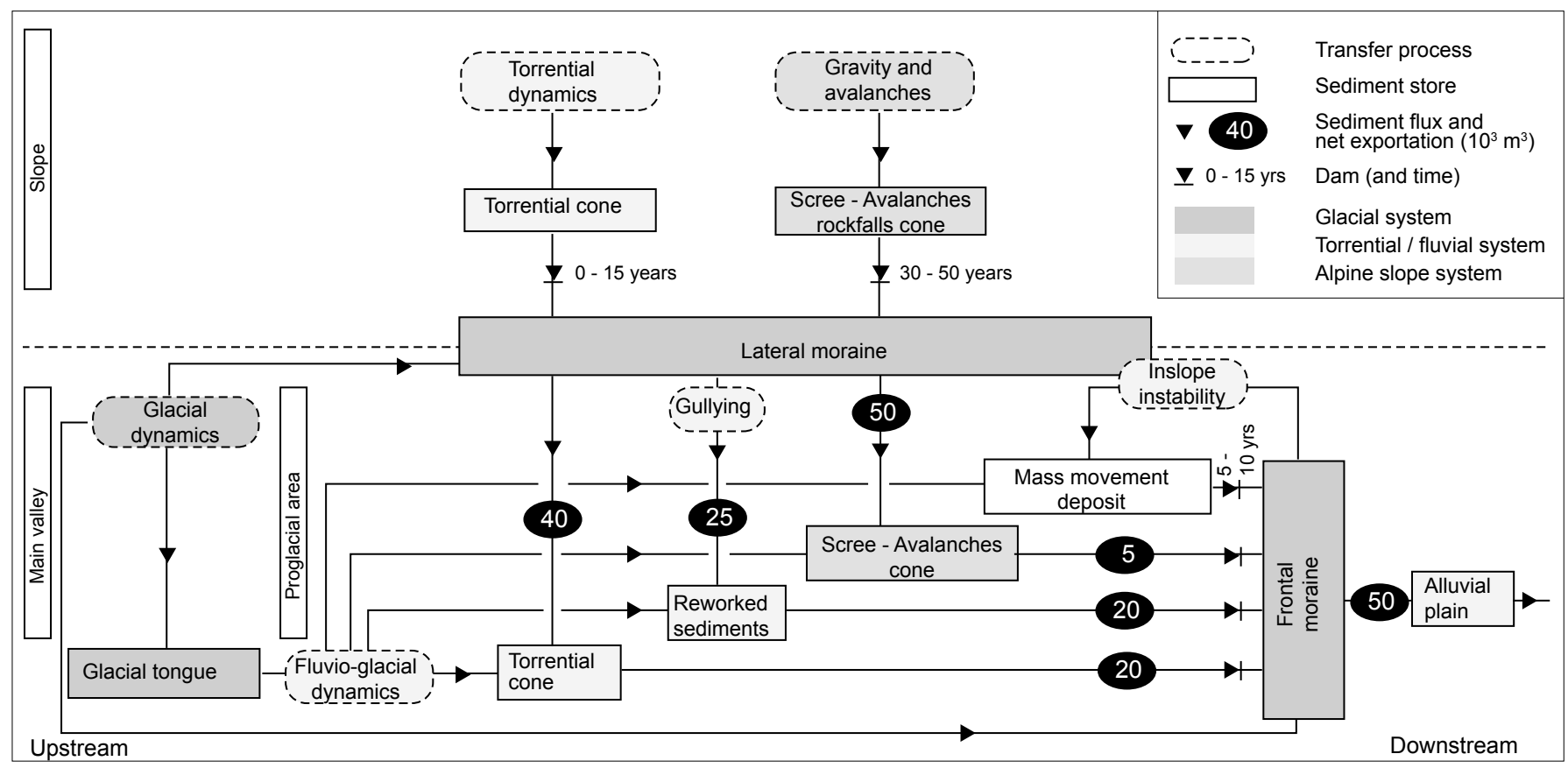

Fig. 10 - Schéma conceptuel du fonctionnement de la cascade sédimentaire de Celse-Nière et bilan sédimentaire depuis le PAG (modifié de Cossart et Fort, 2008).

Fig. 10 - Conceptual scheme of Celse-Nière sediment cascade and sediment budget since the LIA (modified from Cossart and Fort, 2008).

Les flux depuis le PAG sont indiqués dans les disques noirs $\left(10^{3} \mathrm{~m}^{3}\right)$.

Fluxes since LIA are written within dark disks $\left(10^{3} \mathrm{~m}^{3}\right)$ 
alimente chaque nœud du réseau et circule de façon itérative (pas à pas) d'un nœud à l'autre, en suivant les cheminements formalisés par le graphe. Un flux sédimentaire potentiel peut ainsi être simulé tout au long de la cascade sédimentaire étudiée. En complément, ces flux potentiels peuvent permettre de différencier les nœuds, c'est-àdire ceux où s'opère un potentiel engorgement sédimentaire.

Sur le plan algébrique, le débit potentiel $F$ d'un nœud $i$ peut être évalué en considérant le nombre de chemins qui traversent $i$, provenant d'un nœud $j$ en amont et allant jusqu'à l'exutoire $o$. Ce débit est noté $F_{i j 0}$. Cette valeur, pour être correctement interprétée et comparée, doit être normalisée : elle est divisée par le nombre total de chemins qui proviennent de tous les nouds $j$ à $o\left(\mathrm{~F}_{j o}\right)$. Il s'agit ainsi de révéler quelle proportion du flux potentiel total du bassin versant traverse le nœud $i$ (indice de flux, notée $\mathrm{F}_{i}$ ) :

$$
F i=\left(\sum_{i} F_{i j o}\right) /\left(\sum F_{j o}\right)
$$

Des travaux antérieurs (Borselli et al., 2008 ; Cavalli et al., 2013) ont par ailleurs montré qu'un nœud dont la position minimise aussi bien la distance aux sources sédimentaires situées en amont qu'à l'exutoire situé en aval, exerce une plus grande influence sur la cascade sédimentaire. On entend ici par influence non pas la quantité de sédiments transférés, mais l'aptitude à interagir avec les autres nœuds du graphe : il s'agit par exemple de propager un signal, une perturbation, en d'autres points de la cascade sédimentaire (Cossart et Fressard, 2017). La connectivité structurelle peut ainsi être évaluée en considérant l'excentricité des nœuds dans le réseau. L'indice de Shimbel, largement mobilisé dans la description géographique des réseaux de transport (Pumain et Saint-Julien, 2010) a été récemment appliqué aux graphes orientés en géomorphologie (Cossart et Fressard, 2017). Pour un nœud $i$, la valeur de cet indice correspond à la somme de la longueur de tous les chemins reliant tous les autres nœuds $j$ du graphe $\left(d_{i j}\right)$. Pour faciliter les comparaisons à la fois dans l'espace et dans le temps, cet indice est normalisé, c'est-à-dire divisé par la somme de la longueur de tous les chemins dans le réseau, circulant entre tous les nœuds $j$ vers tous les noeuds $k\left(d_{i k}\right)$ :

$$
S h i_{i}=\Sigma d_{i j} / \Sigma d_{j k}
$$

Si l'indice de Shimbel est élevé, alors le nœud contribue à créer de longs chemins dans le réseau (et a donc une position excentrique). Si l'indice de Shimbel est faible, le nœud maximise la compacité du réseau. Il a également été remarqué que l'indice de flux potentiel $F_{i}$ augmente avec l'excentricité $S h i_{i}$ (Cossart et Fressard, 2017). Néanmoins, en raison de la géométrie des liens et $\mathrm{du}$ jeu des confluences entre des sous-cascades sédimentaires, cette relation est marquée par des anomalies, où l'ampleur du flux potentiel apparaît plus élevée ou plus faible que ce que laisserait présager l'emplacement du nœud. Pour estimer cette éventuelle sous ou sur-représentation du volume potentiel de sédiments dans chaque noud, un ratio (noté NSC, indice de connectivité structurelle du réseau) entre le flux potentiel simulé sur un nœud et l'excentricité de ce même nœud a été calculé (Cossart et Fressard, 2017). Plus récemment, le calcul des résidus de l'indice de flux $(R F)$ à partir d'une régression linéaire entre $F_{i}$ et $S h i_{i}$ a été formalisé et offre des résultats prometteurs (Cossart et al., sous-presse).

$$
F_{i}^{\prime}=a S h i_{i}+b
$$

où $F_{i}$ est la valeur théorique attendue de l'indice de débit potentiel compte tenu de la valeur de Shi. Ensuite, les résidus peuvent être calculés.

$$
R F_{i}=F_{i}-F_{i}^{\prime}
$$

Ce calcul est appliqué ici, dans le cadre du bassin versant de Celse-Nière, pour les années 1952, 1981 et 2015. Il s'agit d'explorer le fonctionnement de la cascade sédimentaire et de montrer comment sa capacité à transférer des sédiments a changé.

\subsection{Description de la cascade sédimentaire depuis 1952}

\subsubsection{Structure actuelle de la cascade sédimentaire}

Pour décrire la capacité de la cascade à transférer les sédiments à l'exutoire, nous identifions l'ensemble des entraves (fig. 11), c'est-à-dire les nœuds du graphe qui fonctionnent comme des puits sédimentaires (un seul lien entrant et aucun lien sortant). Ces puits représentent actuellement $14 \%$ du nombre total de nœuds et sont de deux principaux types. Le premier type correspond à des dépôts d'éboulis $(2 \%$ du nombre total de nœuds), situés en pied de versant, et dont la granulométrie dépasse la compétence des cours d'eau proglaciaires. Certains dépôts d'éboulis sont également déconnectés du fond de vallée en raison de la présence de zones tampons comme des roches moutonnées et des terrasses fluvio-glaciaires. Le second type de puits sédimentaires est associé à la présence de moraines (12\% du nombre total de nœuds). En effet, par leur morphologie en forme de crête (jusqu'à plusieurs mètres ou même quelques décamètres de hauteur), les moraines constituent des barrages efficaces à l'écoulement. Les dépôts s'accumulent ainsi contre le barrage morainique. Peu de brèches dans ces édifices autorisent le cheminement sédimentaire : $37 \%$ de la charge sédimentaire potentielle est interceptée par les barrières morainiques.

$\mathrm{Au}$ total, l'ensemble des déconnexions liées aux différents types de puits sédimentaires implique que seulement $61 \%$ de la superficie totale du bassin versant contribue potentiellement à la fourniture sédimentaire à l'exutoire.

\subsection{2. Évolution de la connectivité structurelle depuis 1952}

L'évolution de la structure de la cascade a été reconstituée depuis 1952 (fig. 11A). L'analyse du flux potentiel confirme l'existence d'une fragmentation durable de la cascade sédimentaire par les moraines et les éboulis. En 1952, seulement $8 \%$ du flux potentiel est relié efficacement à l'exutoire. Cette valeur a ensuite progressivement augmenté, atteignant $25 \%$ en 1981 et $61 \%$ actuellement. Cette tendance est essentiellement liée au démantèlement progressif de certains édifices morainiques (latéraux et frontaux) : le nombre de liens déconnectés de la cascade sédimentaire par les crêtes morainiques a diminué de 259 en 1952 à 104 actuellement. A contrario le nombre de dépôts d'éboulis agissant comme pièges a été constant.

Plus précisément, les cartes diachroniques de l'indice $R F$ présentent deux principaux changements locaux affectant la connectivité structurelle, plus particulièrement l'agencement spatial des flux sédimentaires potentiels. Tout d'abord, nous montrons que l'augmentation, entre 1952 et 1981 (fig. 11B), de la zone contribuant à l'alimentation sédimentaire de l'exutoire est due à la reconnexion du sous-bassin du Coup-de-Sabre avec le chenal principal. Un nouveau lien a été créé, en relation avec le développement d'une brèche à travers une moraine qui entravait le torrent proglaciaire de Coup-de-Sabre. Cet événement est intervenu entre 1952 et 1960 d'après l'examen des photographies aériennes. Ensuite, un scénario similaire s'est produit entre 1981 et 2015 (fig. 11C). Deux nouveaux liens ont été créés à travers des 

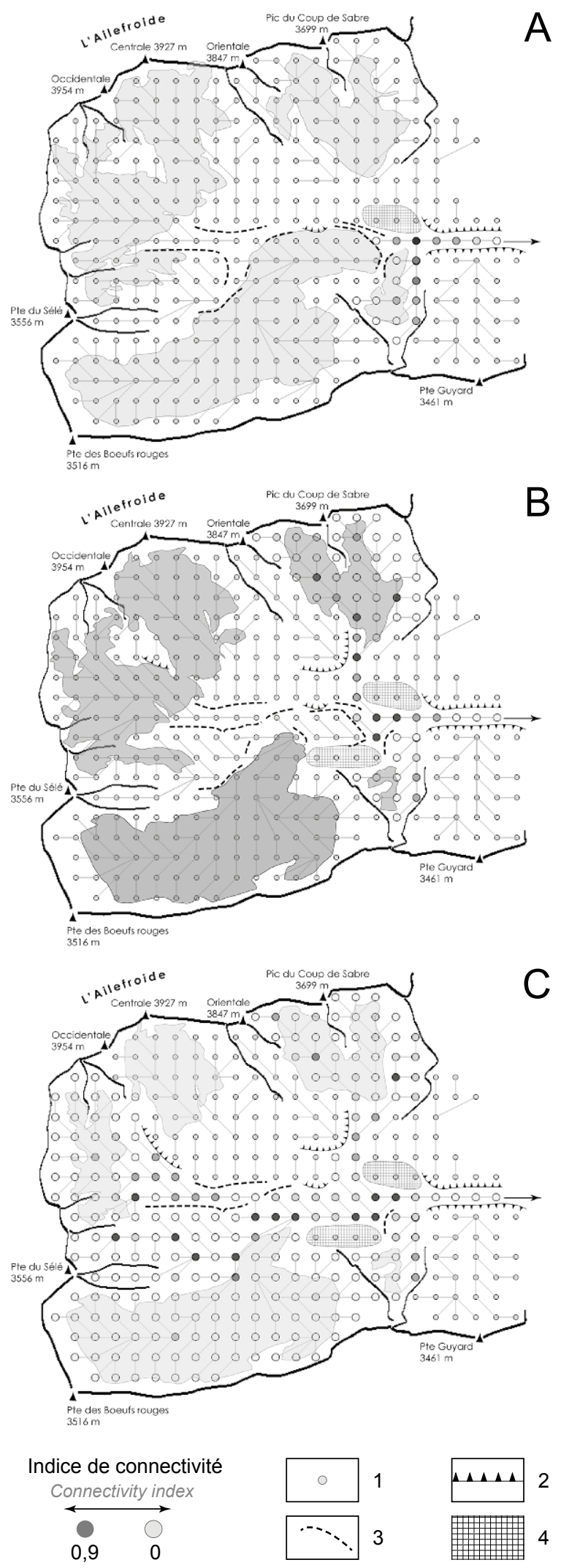

Fig. 11 - Évaluation de la connectivité sédimentaire dans le bassin-versant de Celse-Nière.

A : Structure spatiale de la cascade en 1952 ; B : En 1981;C : En 2015. 1. Déconnecté de l'exutoire; 2 . Tampons ; 3 . Barrières ; 4 . Couvertures.

Fig. 11 - Assessment of connectivity in the Celse-Nière catchment.

A: Spatial structure of the cascade in 1952; B: In 1981; C: In 2015. 1. Disconnected from the outlet; 2. Buffers; 3. Barriers; 4. Blankets édifices morainiques, autorisant la reconnexion des sous-bassins d'Ailefroide et du Sélé. L'incision des crêtes morainiques est intervenue entre 2005 et 2015 d'après les observations de terrain. La création de ces deux seuls liens permet de faire augmenter de $25 \%$ à $61 \%$ la part des nœuds du graphe qui contribuent potentiellement à l'alimentation sédimentaire de l'exutoire.

Ce premier examen de l'évolution du squelette de la cascade sédimentaire montre que l'augmentation de l'efficacité du transfert sédimentaire n'est pas progressive : elle s'effectue par à-coups, rythmés par des reconnexions localisées. La situation des liens créés n'est par ailleurs pas neutre : en autorisant la reconnexion de vastes sous-bassins (vastes eu égard à la dimension totale du bassin-versant étudié), un seul lien peut avoir une incidence globale sur l'ensemble de la cascade. Il s'agit ici d'un exemple de non-linéarité du fonctionnement géomorphologique où une petite cause, locale, peut avoir de grands effets, globaux.

L'indice de connectivité structurelle $R F$ permet d'effectuer une analyse de ces situations stratégiques pour le fonctionnement et l'organisation de la cascade sédimentaire. L'indice le plus élevé était situé en 1981 à la confluence entre le torrent de Coupde-Sabre et le drain principal ; actuellement, il est situé à la confluence entre le torrent d'Ailefroide et le drain principal. Ainsi, la charge sédimentaire potentielle au niveau de ces points est plus élevée que ce que laisserait présager leur situation au sein de la cascade. Dans les deux cas, ces valeurs élevées sont tout d'abord dues aux implications directes de la reconnexion des sous-bassins : 48 nœuds fournissent potentiellement des sédiments à travers la nouvelle liaison créée en aval du Coupde-Sabre en 1981 (fig. 11B), 45 à travers la nouvelle liaison créée en aval du sous-bassin d'Ailefroide en 2015 et, enfin, 108 en aval du sous-bassin de Sélé en 2015 (fig. 11C). En parallèle, ces points névralgiques identifiés par l'indice $R F$ ont une position médiane au sein des longs cheminements sédimentaires qui ont été ouverts en 1981, puis en 2015. Ces longues chaînes au sein du graphe, pour reprendre la terminologie mathématique, ont une longueur qui favorise l'expression des relais de processus géomorphologiques. Concrètement, il s'agit de recueillir des sédiments à partir de nombreuses sources situées en amont, de favoriser ainsi l'émergence d'un signal sédimentaire, et le transmettre en aval vers l'exutoire. Les nœuds situés en position médiane ont ainsi une situation stratégique équilibrée : situés à proximité de multiples sources, dont ils collectent les sédiments pour organiser une pulsation sédimentaire, ils influencent également en aval le signal sédimentaire sur les différents composants de la cascade sédimentaire, jusqu'à l'exutoire.

\section{Discussion}

\subsection{Les interprétations de la connectivité}

Cette revue de la littérature a permis de présenter l'intérêt de la spatialisation et de la mesure de la connectivité écologique à l'aide de graphes paysagers. Comme le défendent d'autres auteurs (Bunn et al., 2000 ; Zetterberg et al., 2010 ; Foltête et al., 2014), le cas d'application présenté montre de quelle manière les graphes paysagers peuvent permettre de répondre à certaines problématiques d'aménagement du territoire ou de conservation. À travers la présentation d'une démarche utilisée en écologie du paysage, nous pouvons identifier un certain nombre de problématiques communes avec les questions des géomorphologues. En effet, formaliser un système sous forme d'un réseau d'interaction amène à interroger comment les agencements de ces interactions confèrent cohérence et 
consistance au réseau, et comment elles rejaillissent directement sur les propriétés de résilience du système. Nous retrouvons ici l'hypothèse commune à toutes les approches mobilisant la notion de connectivité. Que signifie pour autant une forte connectivité ? À l'échelle du réseau entier : est-ce une aptitude du réseau à encaisser/amplifier des forçages extérieurs ? À l'échelle intraréseau : à quoi correspondent les points nodaux, stratégiques, identifiés par leur aptitude à être bien reliés aux autres éléments du réseau ? Quelles implications scientifiques, opérationnelles, peut-on prévoir à partir de ces évaluations de la connectivité ? Si la réponse à ces questions reste débattue, il apparaît toutefois que la connectivité d'un réseau donné se spatialise, se quantifie, qu'il s'agisse d'un réseau écologique ou d'un réseau de flux sédimentaires.

\subsection{Connectivité et suivi diachronique}

D’une manière générale, l'utilisation des métriques de connectivité globales permet de mesurer la connectivité d'un réseau et de la comparer de manière diachronique avant et après la modification du réseau, par évènement naturel ou anthropique. L'interprétation des résultats obtenus est souvent complexe. En effet, il est possible de comparer deux réseaux en évaluant un taux de variation entre deux états. Ces valeurs peuvent permettre d'établir des comparaisons entre plusieurs scénarios, plusieurs types de modifications sur un même réseau. Il est toutefois difficile de savoir dans quelle mesure le réseau est menacé dans sa globalité. Par exemple, il est délicat d'évaluer le seuil de perte de connectivité rendant nécessaire la mise en place de mesures fortes de restauration ou de conservation. De la même façon, les résultats obtenus permettent certes de comparer différentes évolutions d'un même réseau, mais la comparaison entre des réseaux différents reste difficile car les métriques n’ont pas forcément le même ordre de grandeur d'un réseau à l'autre. Comment en effet comparer les valeurs de connectivité absolues d'un réseau très connecté, par exemple dans une zone rurale très boisée, avec celles d'un réseau de zones humides beaucoup plus sporadique ? Comment comparer les valeurs de connectivité mesurées dans deux bassins-versants de taille très différentes? L'une des possibilités est de confronter chaque réseau modélisé à un réseau théorique de référence : un "modèle nul ", dont les propriétés sont déduites des choix qui sont effectués lors de la formalisation initiale. Un bon exemple de "modèle nul » est le réseau planaire idéal, qui maximise le nombre de liens entre les nœuds d'un graphe, et qui sert de référence aux réseaux de transports pour estimer des métriques de connectivité robustes, comparables d'un réseau à un autre (Pumain et Saint-Julien, 2010).

En ce qui concerne les mesures de connectivité locales, les valeurs de la métrique sont mesurées pour chaque nœud du graphe. La comparaison diachronique entre deux états du graphe ne concerne ainsi que les nœuds de chacun des graphes. Ceci implique que les graphes conservent la même structure entre l'état initial et l'état final (après un aménagement par exemple) ce qui n'est pas toujours le cas. Il est alors difficile de savoir à quelle modification du réseau est due la réduction de la connectivité au niveau d'un nœud. En écologie du paysage, la comparaison des réseaux écologiques de plusieurs espèces pose problème. En effet, les taches d'habitats de différentes espèces étant de nature et de taille diverse, il est délicat de comparer différents réseaux d'une même zone d'étude. Dans cette optique, quelques méthodes exploratoires proposées dans Foltête et al. (2014), Bourgeois (2015) et Sahraoui et al. (2017) proposent d'interpoler les métriques locales à l'ensemble de la mosaïque paysagère. De cette manière, chaque pixel de la zone d'étude est caractérisé par une valeur de connectivité, ce qui rend possible les comparaisons de différents réseaux écologiques d'une même zone d'étude. Cette solution soulève d'autres problèmes d'ordre thématique. Que faut-il faire dans les zones présentant des valeurs élevées de connectivité : doit-on favoriser la conservation de ces zones ou peut-on au contraire y autoriser quelques aménagements ? Si la mesure de connectivité est une façon d'explorer le fonctionnement du système, un outil de réflexion, la réponse à ces questions correspond à de futurs champs de recherche.

Les mêmes interrogations existent quant à la bonne interprétation des métriques locales de connectivité en géomorphologie. Le dysfonctionnement de «hotspots", voire leur rupture/déconnexion, peut aboutir à une modification profonde $\mathrm{du}$ système initial, sans présager du caractère finalement péjoratif ou non de cette désorganisation. Au sein d'une cascade sédimentaire, le dysfonctionnement du point ayant la plus haute connectivité amènerait à un scindement en plusieurs systèmes indépendants, de taille aussi équivalente que possible, de telle sorte qu'aucun ne pourrait de toute façon atteindre le niveau d'organisation du système initial (Cossart et Fressard, 2017). On entend ici par niveau d'organisation le jeu des interactions mutuelles qui animent les réseaux. Finalement, ces métriques locales aident à identifier les points les plus névralgiques, c'est-àdire ceux dont le dysfonctionnement aboutirait au maximum de désorganisation du système initial.

\section{Conclusion}

La connectivité apparaît comme un cadre conceptuel qui peut se décliner selon les thématiques propres à diverses disciplines. Dans les systèmes environnementaux développés ici, des flux de natures diverses enregistrent des changements locaux ou globaux : des flux d'espèces ou des flux sédimentaires (tab. 1). Dans chaque cas, le réseau est alors une modalité de formalisation de ces systèmes, et la connectivité une propriété du réseau. Nous avons ici tenu à montrer que la connectivité n'est pas seulement un concept. Elle se mesure, elle peut éventuellement se cartographier. Ce travail d'évaluation nécessite certes d'effectuer des simplifications, et donc des choix, pour formaliser le système étudié. La représentation sous forme de nœuds et de liens en est une, de même que le choix des modalités d'évaluation de la distance au sein du réseau. Une telle simplification, inhérente à tout travail de modélisation, offre toutefois des clefs d'interprétation de l'ensemble des flux qui animent les réseaux. Ainsi la connectivité, si elle ne cherche pas à expliquer l'ensemble de la complexité des systèmes environnementaux, permet de mieux décrypter comment ces systèmes réagissent (ou non) à des forçages globaux (climatiques par exemple) ou locaux (interférences anthropiques). 
Tab. 1 - Spatialiser et mesurer la connectivité à l'aide de la théorie des graphes.

Comparaisons d'exemples d'approches utilisées en écologie du paysage et en géomorphologie.
Tab. 1 - Spatializing and quantifying connectivity by means of graph theory. Comparisons of methods in landscape ecology and geomorphology.

\begin{tabular}{|c|c|c|}
\hline & Écologie du paysage & Géomorphologie \\
\hline Nœuds & $\begin{array}{l}\text { Taches d'habitat d'espèces ou de groupes } \\
\text { d'espèces }\end{array}$ & Réservoirs sédimentaires \\
\hline Liens & Corridors de déplacements & Processus géomorphologiques \\
\hline Matrice & $\begin{array}{l}\text { Classes d'occupation du sol constituant des } \\
\text { zones peu favorables ou défavorables aux } \\
\text { déplacements }\end{array}$ & État de surface \\
\hline Nature du flux & Dispersion intergénérationnelle des espèces & Flux sédimentaire \\
\hline $\begin{array}{l}\text { Métriques globales utilisées dans Tannier et } \\
\text { al. (2016a) et Cossart et Fressard (2017) }\end{array}$ & $\begin{array}{l}\text { PC (Probability of Connectivity) } \\
P C=\frac{\sum_{i=1}^{n} \sum_{j=1}^{n} a_{i} a_{j} p_{i j}^{*}}{A_{L}^{2}} \\
\text { où } a_{i} \text { et } a_{j} \text { sont les surfaces respectives des } \\
\text { taches } i \text { et } j, p_{i j}^{*} \text { le produit maximum des } \\
\text { probabilités de dispersion de tous les } \\
\text { parcours possibles entre la tache } i \text { et } j \text { et } A_{L}^{2} \text { la } \\
\text { surface totale de la zone d'étude. }\end{array}$ & $\begin{array}{l}\text { Nombre de composantes connexes } \\
\text { Sediment delivery ratio : } \\
S D R=V_{h} / V_{o} \\
\text { où } V_{h} \text { est le volume de sédiments apporté par } \\
\text { les sources et où } V_{o} \text { est le volume apporté à } \\
\text { l'exutoire. }\end{array}$ \\
\hline $\begin{array}{l}\text { Métriques locales utilisées dans Bourgeois } \\
\text { (2015) et Cossart et Fressard (2017) }\end{array}$ & $\begin{array}{l}P C_{f l u x}: \\
P C_{f l u x}(j)=\frac{\sum_{i=1}^{n} a_{i} a_{j} p_{i j}^{*}}{A_{L}^{2}} \\
\text { où } a_{i} \text { et } a_{i} \text { sont les surfaces respectives des } \\
\text { taches } i \text { et } j, p_{i j}^{*} \text { le produit maximum des } \\
\text { probabilités de dispersion de tous les } \\
\text { parcours possibles entre la tache } i \text { et } j \text { et } A_{L}^{2} \text { la } \\
\text { surface totale de la zone d'étude. }\end{array}$ & $\begin{array}{l}\text { Accessibilité : } \\
S h i=\Sigma d_{i j} / \Sigma d_{j k} \\
\text { où } d_{i j} \text { est la longueur du chemin reliant un } \\
\text { noeud } i \text { à tous les autres nœuds } j \text { du graphe et } \\
\text { où } d_{j k} \text { est la longueur des chemins entre tous } \\
\text { les nœuds } j \text { vers tous les nœuds } k \text {. } \\
\mathbf{R F}(\text { residual flow }): \\
R F_{i}=F_{i} \text { - }\left(a \text { Shi } i_{i}+b\right) \\
\text { où } F_{i} \text { est le volume potentiel de sédiments } \\
\text { transitant dans un noeud } i, \text { Shi } i_{i} \text { est l'indice } \\
\text { d'accessibilité de Shimbel, aet } b s o n t \text { les } \\
\text { paramètres de la régression linéaire entre } F_{i} \\
\text { et } S h i_{i}\end{array}$ \\
\hline Interprétation/signification de la métrique & $\begin{array}{l}\text { Mesure la probabilité que des individus } \\
\text { placés au hasard dans la zone d'étude se } \\
\text { situent dans des taches d'habitat connectées } \\
\text { à un niveau global }(\mathrm{PC}) \text {. La métrique locale } \\
P C_{f l u x} \text { mesure cette probabilité à l'échelle } \\
\text { d'une tache d'habitat. La somme des valeurs } \\
\text { de la métrique } P C_{f u x} \text { de chaque tache } \\
\text { d'habitat correspond à la valeur globale de la } \\
\text { métrique PC. }\end{array}$ & $\begin{array}{l}\text { «Hotspots of change ». Il s'agit d'identifier } \\
\text { les nœuds qui ont une situation stratégique } \\
\text { et peuvent ainsi influencer le } \\
\text { fonctionnement de multiples compartiments } \\
\text { d'une cascade sédimentaire. }\end{array}$ \\
\hline
\end{tabular}

Aide à la décision en aménagement du territoire

Préserver la connectivité :

- comparaison de l'impact potentiel de plusieurs projets

- aide à l'élaboration de Trames Vertes et

Bleues

Applications potentielles
- identification de zones clés pour maintenir ou restaurer la connectivité (création de mares, de haies, passages à faune)

- évaluation des impacts d'un projet pour la mise en place de mesures de compensation

Casser la connectivité:

- minimiser la pullulation d'espèces invasives

comme le campagnol terrestre
Aide à la décision en gestion de

l'environnement

Préserver la connectivité:

- restauration des continuités hydrosédimentaires

- Désaménagement des hydrosystèmes

- Réflexion sur les effacements d'ouvrages

hydrauliques à effectuer en priorité

Casser la connectivité:

- Casser la connectivité pour calmer des processus érosifs notamment en contexte d'érosion des sols cultivés 


\section{Références}

Adriaensen F., Chardon J.P., De Blust G., Swinnen E., Villalba S., Gulinck H., Matthysen E. (2003) - The application of 'least-cost' modelling as a functional landscape model. Landscape and Urban Planning, 64 (4), 233-247.

DOI : $10.1016 / \mathrm{s} 0169-2046(02) 00242-6$

Apicella C.L., Marlowe F.W., Fowler J.H., Christakis N. (2012) Social Networks and Cooperation in Hunter-Gatherers. Nature, 481 (7382), 497-501.

DOI : $10.1038 /$ nature 10736

Bak P., Tang C., Wiesenfeld K. (1987) - Self-organized criticality: An explanation of the 1/f noise. Physical Review Letters, 59, 381-384.

DOI : 10.1103/physrevlett.59.381

Bak P., Tang C., Wiesenfeld K. (1988) - Self-organised criticality. Physical Review A., 38 (1), 364-374.

DOI : 10.1103/PhysRevLett.59.381

Beier P., Noss R.F. (1998) - Do habitat corridors provide connectivity? Conservation Biology, 12 (6), 1241-1252. DOI : $10.1111 / \mathrm{j} .1523-1739.1998 .98036 . x$

Belisle M. (2005) - Measuring landscape connectivity: the challenge of behavioral landscape ecology. Ecology, 86, 1988-1995.

DOI : 10.1890/04-0923

Bennett G. (2004) - Integrating biodiversity conservation and sustainable use: lessons learned from ecological networks. World Conservation Union (IUCN), Gland, Switzerland, and Cambridge, UK, $55 \mathrm{p}$.

Borselli L., Cassi P., Torri D. (2008) - Prolegomena to sediment and flow connectivity in the landscape: a GIS and field numerical assessment. Catena, 75, 268-277.

DOI : $10.1016 /$ j.catena.2008.07.006

Bourgeois M. (2015) - Impacts écologiques des formes d'urbanisation. Modélisations urbaines et paysagères. Thèse de doctorat, Université de Franche-Comté, 369 p.

Bunn A.G., Urban D.L., Keitt T.H. (2000) - Landscape connectivity: A conservation application of graph theory. Journal of Environmental Management, 59 (4), 265-278.

DOI : $10.1006 /$ jema.2000.0373

Caine N. (1984) - Elevational contrasts in contemporary geomorphic activity in the Colorado Front Range. Studia Geomorphologica Carpatho-Balcanica, 18, 5-31.

Calabrese J.M., Fagan W.F. (2004) - A comparison-shopper's guide to connectivity metrics. Frontiers in Ecology and the Environment, 2 (10), 529-536.

DOI : $10.2307 / 3868383$

Cavalli M., Trevisani S., Comiti F., Marchi L. (2013) Geomorphometric assessment of spatial sediment connectivity in small Alpine catchments. Geomorphology, 188, 31-41. DOI : 10.1016/j.geomorph.2012.05.007

Clauzel C., Girardet X., Foltête J.C. (2013) - Impact assessment of a high-speed railway line on species distribution: application to the European tree frog (Hyla arborea) in Franche-Comté. Journal of environmental management, 127, 125-134.

DOI : 10.1016/j.jenvman.2013.04.018

Cole J.P., King C.A.M. (1968) - Quantitative Geography. Techniques and Theories in Geography. Wiley, Londres, $692 \mathrm{p}$.

Cossart E. (2013) - Influence of local vs regional settings on glaciation pattern in the French Alps. Geografia Fisica e Dinamica Quaternaria, 36 (1), 39-52.

DOI : $10.4461 /$ GFDQ.2013.36.3
Cossart E. (2016) - L'(in)efficacité géomorphologique des cascades sédimentaires en question. Les apports d'une analyse réseau. Cybergeo. European Journal of Geography, 778, DOI : $10.4000 /$ cybergeo. 27625 .

Cossart E., Fort M. (2008) - Sediment release and storage in early deglaciated areas: Towards an application of the exhaustion model from the case of Massif des Écrins (French Alps) since the Little Ice Age. Norsk Geografisk Tidsskrift-Norwegian Journal of Geography, 62, 115-131.

DOI : $10.1080 / 00291950802095145$

Cossart E., Fressard M. (2017) - Assessment of structural connectivity within catchment: insights from graph theory. Earth Surface Dynamics, 5, 253-268.

DOI : 10.5194/esurf-2016-55

Cossart E., Viel V., Lissak C., Reulier R., Fressard M., Delahaye D. (sous-presse) - How might sediment connectivity change in space and time? Land degradation and development.

Dale M.R.T., Fortin M.J. (2010) - From Graphs to Spatial Graphs. Annual Review of Ecology, Evolution, and Systematics, 41 (1), 21-38.

DOI : 10.1146/annurev-ecolsys-102209-144718

Delahaye D., Guermond Y., Langlois P. (2002) - Spatial interaction in the run-off process. Cybergeo: European Journal of Geography, 213, 1-13.

DOI : 10.4000/cybergeo.3795

Douvinet J., Delahaye D., Langlois P. (2008) - Modélisation de la dynamique potentielle d'un bassin versant et mesure de son efficacité structurelle. Cybergeo: European Journal of Geography, $412,1-22$.

DOI : $10.4000 /$ cybergeo. 16103

Faulkner H. (2008) - Connectivity as a crucial determinant of badland geomorphology and evolution. Geomorphology, 100 (1-2), 91-103.

DOI : 10.1016/j.geomorph.2007.04.039

Ferguson R.I. (1981) - Channel forms and channel changes. In Lewin J. (Ed.), British Rivers, Allen and Unwin, London: 90-125.

Freeman L.C. (1979) - Centrality in social networks conceptual clarification. Social Networks, 1 (3), 215-239.

DOI : 10.1016/0378-8733(78)90021-7

Foltête J.C., Clauzel C., Vuidel G. (2012) - A software tool dedicated to the modelling of landscape networks. Environmental Modelling \& Software, 38, 316-327.

DOI : $10.1016 /$ j.envsoft.2012.07.002

Foltête J.C., Girardet X., Clauzel C. (2014) - A methodological framework for the use of landscape graphs in land-use planning. Landscape and Urban Planning, 124, 140-150. DOI : 10.1016/j.landurbplan.2013.12.012

Forman R.T.T., Godron M (1986) - Landscape ecology. J. Wiley \& sons, New York, 619 p.

Fryirs K., Brierley G.J., Preston N.J., Kasai M. (2007) - The disconnectivity of catchment-scale sediment cascades. Catena, 70, 49-67.

DOI : 10.1002/esp.3242

Fu W., Liu S., Degloria S.D., Dong S., Beazley R. (2010) Characterizing the "fragmentation-barrier " effect of road networks on landscape connectivity: A case study in Xishuangbanna, Southwest China. Landscape and Urban Planning, 95 (3), 122-129.

DOI : 10.1016/j.landurbplan.2010.06.005 
Galpern P., Manseau M., Fall A. (2011) - Patch-based graphs of landscape connectivity: A guide to construction, analysis and application for conservation. Biological Conservation, 144 (1), 44-55.

DOI : 10.1016/j.biocon.2010.09.002

García-Feced C., Saura S., Elena-Rosselló R. (2011) - Improving landscape connectivity in forest districts: A two-stage process for prioritizing agricultural patches for reforestation. Forest Ecology and Management, 261 (1), 154-161.

DOI : $10.1016 /$ j.foreco.2010.09.047

Girardet X., Foltête J.C., Clauzel C. (2013) - Designing a graph-based approach to landscape ecological assessment of linear infrastructures. Environmental Impact Assessment Review, 42, 10-17.

DOI : 10.1016/j.eiar.2013.03.004

Gleyze J.-F. (2008) - Effets spatiaux et effets réseau dans l'évaluation d'indicateurs sur les nœuds d'un réseau d'infrastructure. Cybergeo : European Journal of Geography, article 370.

DOI : $10.4000 /$ cybergeo. 5532

Gurrutxaga M., Rubio L., Saura S. (2011) - Key connectors in protected forest area networks and the impact of highways: A transnational case study from the Cantabrian Range to the Western Alps (SW Europe). Landscape and Urban Planning, 101 (4), 310-320.

DOI : 10.1016/j.landurbplan.2011.02.036

Haggett P., Chorley R.J. (1969) - Network analysis in geography. London Edward Arnold, Science, 348 p.

Hanski I., Gilpin M. (1991) - Metapopulation dynamics: brief history and conceptual domain. Biological Journal of the Linnean Society, 42 (1), 3-16.

DOI : $10.1111 / j .1095-8312.1991 . t b 00548 . x$

Hardy A.C. (1924) - The herring in relation to its animate environment, part 1. Ministry of agriculture and fisheries, 7 (3), $1-53$.

Harvey A.M. (2001) - Coupling between hillslopes and channels in upland fluvial systems. implications for landscape sensitivity, illustrated from the Howgill Fells, northwest England. Catena, 42, 225-250.

DOI : 10.1016/s0341-8162(00)00139-9

Heckmann T., Schwanghart W. (2013) - Geomorphic coupling and sediment connectivity in an alpine catchment - exploring sediment cascades using graph theory. Geomorphology, 182, 89-103.

DOI : 10.1016/j.geomorph.2012.10.033

Heckmann T., Schwanghart W., Phillips J.D. (2015) - Graph theory. Recent developments of its application in geomorphology. Geomorphology, 243, 130-146.

DOI : 10.1016/j.geomorph.2014.12.024

Heckmann T., Vericat D. (2018) - Computing spatially distributed sediment delivery ratios: Inferring functional sediment connectivity from repeat high-resolution Digital Elevation Models. Earth Surface Processes and Landforms, in press. DOI : $10.1002 /$ esp.4334

Hess G.R., Fischer R.A. (2001) - Communicating clearly about conservation corridors. Landscape and Urban Planning 55 (3), 195-208.

DOI : 10.1016/s0169-2046(01)00155-4

Hoffmann T. (2015) - Sediment residence time and connectivity in non-equilibrium and transient geomorphic systems. EarthScience Reviews, 150, 609-627.

DOI : 10.1016/j.earscirev.2015.07.008
Ims R., Yoccoz N. (1997) - Studying transfer processes in metapopulation: emigration, migration, and colonization. In Hanski I., Gilpin M. (Eds), Metapopulation Biology: Ecology, Genetics, and Evolution, Academic Press, 247-265.

DOI : $10.1016 /$ b978-012323445-2/50015-8

Jäckli H. (1957) - Gegenwartsgeologie des bündnerischen Rheingebietes Beiträge zur Geologie der Schweiz, Geotechnische Serie, $36,126 \mathrm{p}$.

DOI : $10.1177 / 0309133306071958$

Johnson M., Gaines M. (1985) - Selective basis for emigration of the prairie vole, Microtus ochrogaster: open field experiment. Journal of Animal Ecology, 54, 399-410. DOI : $10.2307 / 4487$

Lindenmayer D.B., Margules C.R., Botkin D.B. (2000) - Indicators of Biodiversity for Ecologically Sustainable Forest Management. Conservation Biology, 14 (4), 941-950.

DOI : $10.1046 /$ j.1523-1739.2000.98533.x

Lookingbill T.R., Gardner R.H., Ferrari J.R., Keller C.E. (2010) Combining a dispersal model with network theory to assess habitat connectivity. Ecological Applications, 20 (2), 427-441.

DOI : 10.1890/09-0073.1

McRae B.H., Dickson B.G., Keitt T.H., Shah V.B. (2008) - Using circuit theory to model connectivity in ecology, evolution, and conservation. Ecology, 89 (10), 2712-2724.

DOI : $10.1890 / 07-1861.1$

Merriam G. (1984) - Connectivity: a fundamental ecological characteristic of landscape pattern. In Brandt J., Agger P. (Eds), Methodological Landscape Ecology Research Planning proceedings, Roskilde University Centre, 5-15.

May R.M. (1973) - Stability and complexity in model ecosystems. Princeton University Press, Princeton, 304 p.

Meunier M. (1991) - Eléments d'hydraulique torrentielle. Grenoble, CEMAGREF, $282 \mathrm{p}$.

Minor E.S., Urban D.L. (2007) - Graph theory as a proxy for spatially explicit population models in conservation planning. Ecological Applications, 17 (6), 1771-1782. DOI : $10.1890 / 06-1073.1$

Newman M. (2010) - Networks: an introduction. Oxford University Press. 775 p.

DOI : 10.1093/acprof:oso/9780199206650.001.0001

Opdam P., Foppen R., Vos C.C. (2002) - Bridging the gap between empirical knowledge and spatial planning in landscape ecology. Landscape Ecology, 16, 767-779.

DOI : 10.1023/A:1014475908949

Opdam P., Pouwels R., Van Rooij S., Steingröver E., Vos C.C. (2008) - Setting biodiversity targets in participatory regional planning: Introducing ecoprofiles. Ecology and Society, 13 (1), $20 \mathrm{p}$.

DOI : $10.5751 /$ es-02438-130120

Östborn P., Gerding H. (2014) - Network analysis of archaeological data: a systematic approach. Journal of Archaeological Science, $46,75-88$. DOI : $10.1016 /$ j.jas.2014.03.015

Pumain D., Saint-Julien T. (2010) - Analyse spatiale : les localisations. Cursus, Armand Colin, Paris, 192 p.

Rapp A. (1960) - Recent development of mountain slopes in Kärkevagge and surroundings, northern Scandinavia. Geografiska Annaler, 42, 65-200.

DOI : $10.1080 / 20014422.1960 .11880942$

Rayfield B., Fortin M.J., Fall A. (2011) - Connectivity for conservation: a framework to classify network measures. Ecology, $92(4), 847-858$.

DOI : $10.1890 / 09-2190.1$ 
Rodrigue J.P. (2017) - Geography of transport system. New-York, Routledge, $441 \mathrm{p}$.

Sahraoui Y., Foltête J.C., Clauzel C. (2017) - A multi-species approach for assessing the impact of land-cover changes on landscape connectivity. Landscape Ecology, 32 (9), 1819-1835. DOI : 10.1007/s10980-017-0551-6

Saura S., Pascual-Hortal L. (2007) - A new habitat availability index to integrate connectivity in landscape conservation planning: Comparison with existing indices and application to a case study. Landscape and Urban Planning, 83 (2-3), 91-103. DOI : 10.1016/j.landurbplan.2007.03.005

Sordello R., Comolet-Tirman J., De Massary J., Dupont P., Haffner P., Rogeon G., Siblet J.P., Touroult J., Trouvilliez J. (2011) - Trame verte et bleue - Critères nationaux de cohérence Contribution à la définition du critère sur les espèces. MNHNSPN, $57 \mathrm{p}$.

Tannier C., Foltête J.C., Girardet X. (2012) - Assessing the capacity of different urban forms to preserve the connectivity of ecological habitats. Landscape and Urban Planning, 105 (1), 128-139.

DOI : 10.1016/j.landurbplan.2011.12.008

Tannier C., Bourgeois M., Houot H., Foltête J.C. (2016a) - Impact of urban developments on the functional connectivity of forested habitats: a joint contribution of advanced urban models and landscape graphs. Land Use Policy, 52, 76-91.

DOI : 10.1016/j.landusepol.2015.12.002

Tannier C., Hirtzel J., Stephenson R., Couillet A., Vuidel G., Youssoufi S. (2016b) - Conception and use of an individualbased model of residential choice in a planning decision process. Feedback from an experimental trial in the city of Besançon, France. Progress in Planning, 108, 1-38.

DOI : 10.1016/j.progress.2015.04.001

Taylor P.D., Fahrig L., Henein K., Merriam G. (1993) Connectivity Is a Vital Element of Landscape Structure. Oikos, 68 (3), 571-573.

DOI : $10.2307 / 3544927$
Tischendorf L., Fahrig L. (2000) - How should we measure landscape connectivity? Landscape Ecology, 15, 633-641. DOI : 10.1023/A:1008177324187

Urban D.L., Keitt T. (2001) - Landscape Connectivity : A GraphTheoretic Perspective. Ecology, 82 (5), 1205-1218. DOI : $10.2307 / 2679983$

Urban D.L., Minor E.S., Treml E.A., Schick R.S. (2009) - Graph models of habitat mosaics. Ecology letters, 12 (3), 260-273. DOI : $10.1111 / \mathrm{j} .1461-0248.2008 .01271 . x$

Vasas V., Magura T., Jordán F., Tóthmérész B. (2009) - Graph theory in action: evaluating planned highway tracks based on connectivity measures. Landscape Ecology, 24 (5), 581-586. DOI : 10.1007/s10980-009-9346-8

Vos C., Verboom J., Opdam P., Ter Braak C. (2001) - Toward ecologically scaled landscape indices. The American Naturalist, 157 (1), 24-41. DOI : $10.2307 / 3079086$

Walker R.G. (1990) - Perspective, facies modelling and sequence stratigraphy. Journal of Sedimentary Petrology, 60, 777-786. DOI : 10.1306/212F926E-2B24-11D7-8648000102C1865D

Whallon R. (2006) - Social networks and information: Non"utilitarian" mobility among hunter-gatherers. Journal of Anthropological Archaeology, 25, 259-270.

DOI : 10.1016/j.jaa.2005.11.004

Wiens J.A., Schooley R.L., Weeks R.D. (1997) - Patchy Landscapes and Animal Movements: Do Beetles Percolate? Oikos, 78 (2), 257-264.

DOI : $10.2307 / 3546292$

Zetterberg A., Mörtberg U.M., Balfors B. (2010) - Making graph theory operational for landscape ecological assessments, planning, and design. Landscape and Urban Planning, 95 (4), 181-191.

DOI : 10.1016/j.landurbplan.2010.01.002

\section{Abridged English version}

Connectivity is considered a key for understanding how environmental systems respond to climate change and land-use change. First, it helps at studying the unsteadiness of environmental systems, especially in ecology and geomorphology. In ecology movement of individuals, populations, and communities within a network of habitats and landscapes (fig. 1) is highly variable, complex, depending on both structure of the network and local influences of habitats surroundings (fig. 2). Such a complex behaviour is also observed in geomorphic signals, especially sediment delivery, that cannot be interpreted easily (e.g. in terms of climate change, anthropogenic influences, etc.). Many authors exhibited a "sedimentological anarchy" at catchment scale because geomorphic processes may be coupled to create a sediment impulse, or may be antagonistic to create a blockage, alternately.

The main originality of this paper is to couple the knowledge from two main field of research within environmental sciences: ecology and geomorphology. Recent developments have been led independently in these both field of research, so that we want to share and cross the expertise to develop innovative methods to assess connectivity (fig. 3). In geomorphology and in ecology, we emphasize a common objective: understanding how processes at local scales may be combined to understand the functioning of the whole environmental system. Such a multiscalar framework distinguishes the coupling of processes and connectivity. On the one hand coupling refers to elementary interactions at the relatively small scale. On the other hand the degree of coupling refers to the combined effect of various linkages between system components and is termed to "connectivity". In ecology, shifting from the local to the broader scale remains a main issue to well explain how loss of linkages on landscape scales (tens of square kilometres) also has consequences at regional and international scales (hundreds of square kilometres and more), then on the potential extinction due to anthropogenic impacts and climate change. In geomorphology, shifting from the local to the catchment scale remains an issue to well explain how small-scale measurements of erosion result in broad-scale patterns and processes.

In details, this paper first exemplifies the development of numerical methods to draw exhaustive inventory of ecological landscape units, in the urban area of Besançon (eastern France) (fig. 5) and then how all the local linkages may create complex interactions within the environmental systems (i.e. ecological network) (fig. 6). In a second step, we exemplify how connectivity can be assessed in a catchment to exhibit to what extent the ability of a sediment cascade emerges from a complex set of local interactions in the mountain range of Écrins (French Alps) (fig. 4; fig. 9). These methods are based upon the application of graph theory. Graph theory offers mathematical tools to analyse statistically and spatially the assemblages of all the components 
of an environmental system. The example developed here seeks at evaluating the ecological impact of different patterns of urbanization on an ecological network (fig. 7; fig. 8). Some recent developments are quite similar in geomorphology. In both cases the methodological framework of connectivity analysis focuses on the structural connectivity, i.e. the influence of the spatial patterns drawn by the linkages, within both ecological network and sediment cascades (fig. 10; fig. 11). In details, connectivity indices will reveal how influent is a node within a sediment cascade, and within an ecological network (tab. 1). In each type of network, high connectivity nodes are the ones where a disruption would imply the more dramatic damages on the network functioning. 